\title{
Hepatic stellate cells contribute to progenitor cells and liver regeneration
}

\author{
Claus Kordes, ${ }^{1}$ Iris Sawitza, ${ }^{1}$ Silke Götze, ${ }^{1}$ Diran Herebian, ${ }^{2}$ and Dieter Häussinger ${ }^{1}$ \\ ${ }^{1}$ Clinic of Gastroenterology, Hepatology and Infectious Diseases, and ²Department of Ceneral Pediatrics, Neonatology and Pediatric Cardiology, Heinrich Heine University, Düsseldorf, Germany.
}

\begin{abstract}
Retinoid-storing hepatic stellate cells (HSCs) have recently been described as a liver-resident mesenchymal stem cell (MSC) population; however, it is not clear whether these cells contribute to liver regeneration or serve as a progenitor cell population with hepatobiliary characteristics. Here, we purified HSCs with retinoid-dependent fluorescence-activated cell sorting from eGFP-expressing rats and transplanted these GFP ${ }^{+}$HSCs into wild-type (WT) rats that had undergone partial hepatectomy in the presence of 2-acetylaminofluorene (2AAF) or retrorsine, both of which are injury models that favor stem cell-based liver repair. Transplanted HSCs contributed to liver regeneration in host animals by forming mesenchymal tissue, progenitor cells, hepatocytes, and cholangiocytes and elevated direct bilirubin levels in blood sera of GUNN rats, indicating recovery from the hepatic bilirubin-handling defect in these animals. Transplanted HSCs engrafted within the bone marrow (BM) of host animals, and HSC-derived cells were isolated from BM and successfully retransplanted into new hosts with injured liver. Cultured HSCs transiently adopted an expression profile similar to that of progenitor cells during differentiation into bile acid-synthesizing and -transporting hepatocytes, suggesting that stellate cells represent a source of liver progenitor cells. This concept connects seemingly contradictory studies that favor either progenitor cells or MSCs as important players in stem cell-based liver regeneration.
\end{abstract}

\section{Introduction}

Hepatic stellate cells (HSCs) are characterized by exceptionally high amounts of retinoids in their quiescent state. These are mainly stored as retinyl palmitate in membrane-coated lipid vesicles and exhibit characteristic retinoid fluorescence after excitation by UV light. HSCs are primarily known as collagen-producing cells in the liver, which are responsible for fibrogenesis in chronic liver diseases (1). Despite intensive research for decades, HSCs have remained enigmatic (2). For instance, stellate cells express molecular markers of different germ layers, which impede a clear statement about their origin (3). The presence of glial fibrillary acidic protein (GFAP) and many other neuroectodermal proteins in stellate cells (4) led to the view that they may derive from the neural crest. This concept has been questioned by microscopic as well as fate-mapping analysis in the developing liver, which indicated that stellate cells apparently originate from mesenchymal cells located in the subendothelial space of the septum transversum $(5,6)$. This view is supported by the expression of mesodermal marker proteins such as desmin and $\alpha$-smooth muscle actin ( $\alpha$-SMA or actin $\alpha 2$ /ACTA2), especially in activated HSCs (7).

Until recently, HSCs were mainly studied with regard to their fibrogenic potential in chronic disease, whereas their identity and function in normal liver received little attention. Recent data suggest that stellate cells represent liver-resident mesenchymal stem cells (MSCs) due to their MSC-related expression profile, their

\section{Related Commentary: p. 5099}

Conflict of interest: The authors have declared that no conflict of interest exists Submitted: June 23, 2014; Accepted: September 11, 2014.

Reference information: J Clin Invest. 2014;124(12):5503-5515. doi:10.1172/JCI74119. potential to differentiate into adipocytes or osteocytes, and their supportive effects on extramedullary hematopoiesis $(8,9)$. In line with this, stellate cells can originate from the bone marrow (BM) $(10,11)$, where MSCs were initially discovered (12), and reside in the liver close to endothelial cells, as is the case for MSCs in other organs (13). Quiescent stellate cells are typically located between sinusoidal endothelial cells and hepatocytes in the space of Disse, which has characteristics of stem cell niches $(14,15)$. Moreover, activated stellate cells can develop into hepatocyte-like cells in vitro $(14,16)$, and fate-mapping experiments using Gfap and Acta2 promoters indicate that they contribute to liver regeneration in vivo (17-19). In contrast to this, lineage-tracing experiments with lecithin retinol acyltransferase (LRAT) and mesoderm posterior 1 homolog (MESP1) failed to demonstrate a differentiation of HSCs into epithelial cells during regeneration of the injured mouse liver (20, 21). Interestingly, LRAT and MESP1, which are suggested to be specifically expressed by HSCs or their precursors $(20,21)$, are expressed by embryonic and adult stem cells from mice and humans (Embryonic Stem Cell Database, http://biit.cs.ut.ee/ escd/; refs. 22, 23). Owing to these opposing lineage-tracing studies, we performed cell transplantation experiments with HSCs in the present study to determine their contribution to liver regeneration. Transplanted stellate cells from the pancreas were recently reported to participate in liver repair through differentiation into epithelial cell lineages in a stem cell-based in vivo liver regeneration model (24). Also, MSCs from the BM and adipose tissue were shown to differentiate into liver parenchymal cells (25-27). Tissue-specific engraftment (homing), trafficking to sites of organ injury, and participation in tissue repair are important properties of stem cells that can be analyzed by cell transplantation experiments $(28-31)$. Stem cells can be repeatedly transplanted into 

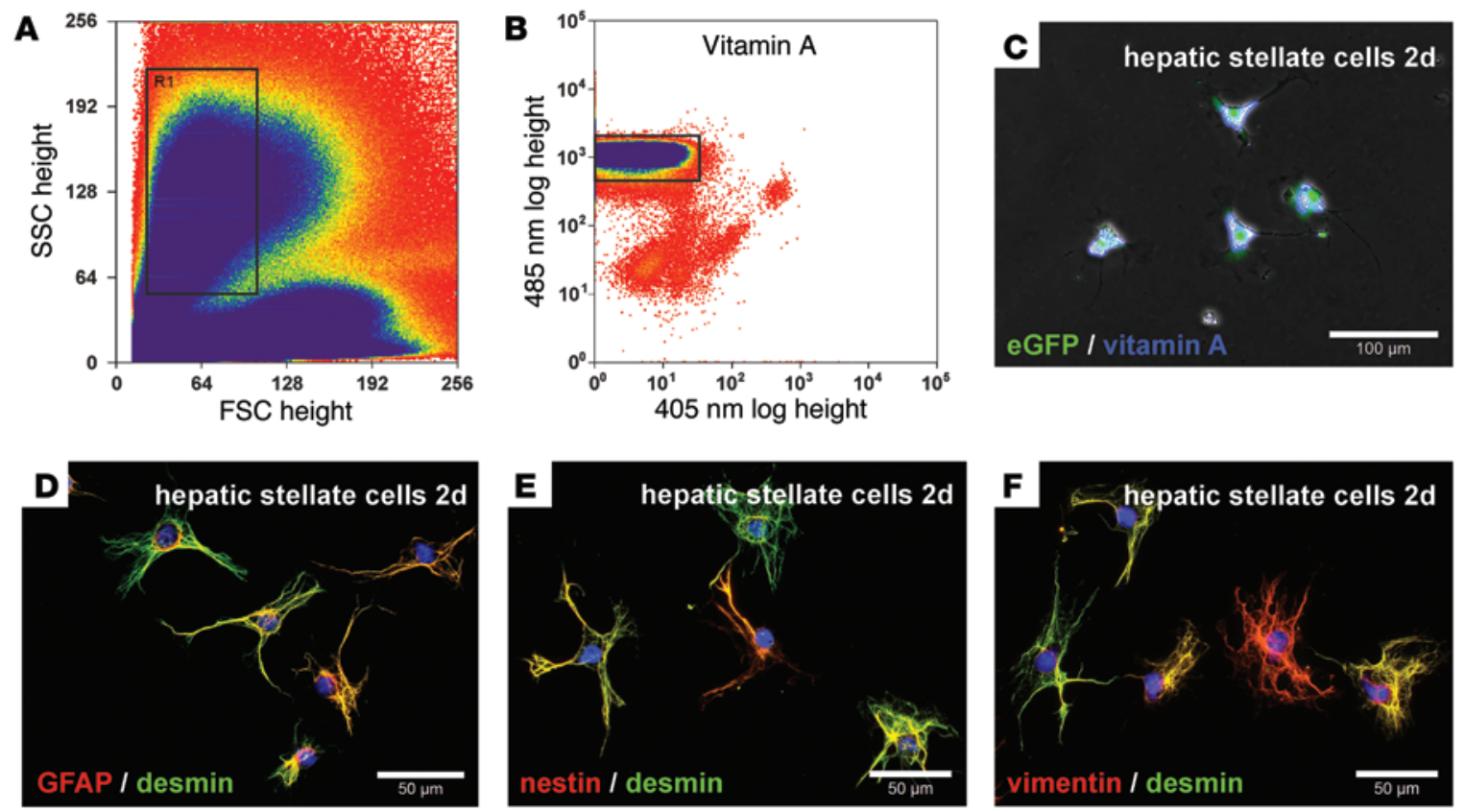

Figure 1. Characterization of freshly isolated HSCs from eGFP+ rats sorted by retinoid-dependent FACS. HSCs were enriched by density gradient centrifugation and further purified by FACS using (A) forward (FSC) and side scatter (SSC) as well as (B) retinoid fluorescence. HSCs with (A) similar morphological characteristics (gate R1) were (B) further analyzed for their retinoid fluorescence by UV light excitation. (B) HSCs with intense retinoid fluorescence (gate) were (C) cultured for 2 days and exhibited retinoid (blue) and eGFP (green) fluorescence as assessed by blue and UV light excitation of living cells. HSCs sorted by FACS expressed (D) GFAP, (E) nestin, and (F) vimentin (red), along with (D-F) desmin (green), as determined by immunofluorescence. Cell nuclei were stained with DAPI (blue). Scale bars: $100 \mu \mathrm{m}$ (C); $50 \mu \mathrm{m}$ (D-F).

different organisms, and their retransplantation capacity is considered to represent one of their key features (29). Cell transplantation studies are therefore required to further validate stem cell characteristics of HSCs. In view of the generation of hepatocytes from HSCs, the question arises whether established liver progenitor cells, which are called oval cells in rodents, could represent an intermediate stage during hepatic differentiation of stellate cells or other MSCs. Liver progenitor cells are small cells that transiently appear after severe liver injury to restore liver mass when the proliferation of hepatocytes is impaired $(32,33)$. These progenitor cells are identified by the expression of epithelial cell adhesion molecule (EPCAM), keratin 19 (K19), and $\alpha$-fetoprotein and protrude from the portal field into the injured liver tissue by forming duct-like structures (ductular reaction). Liver progenitor cells are reported to originate from stem cells with epithelial characteristics (34), but they also show features of mesodermal cells, such as the expression of vimentin (35). The present study addresses whether progenitor cells can derive from MSC populations such as hepatic stellate cells with mesodermal characteristics. The data show that transplanted HSCs can home to the injured liver and contribute to tissue regeneration by developing into putative progenitor cells and epithelial as well as mesenchymal tissues. In this process, progenitor cells seem to represent an intermediate stage of differentiating HSCs and other MSC populations.

\section{Results}

Characterization of isolated HSCs. HSCs from male $\mathrm{eGFP}^{+}$Wistar rats were isolated by enzymatic perfusion of the liver and subsequently enriched by Nycodenz density gradient centrifugation.
Owing to their high lipid content, HSCs formed a clear layer at the upper Nycodenz gradient after centrifugation (not shown). HSCs enriched by this technique were then sorted by retinoiddependent fluorescence-activated cell sorting (FACS). During FACS analysis, we determined the morphology of HSCs by forward and side scatter (Figure 1A). HSCs with similar morphology (Figure 1A, gate) and characteristic retinoid (vitamin A) fluorescence after excitation at $350 \mathrm{~nm}$ (Figure 1B, gate) were finally collected. We found that HSCs were about $13.2 \pm 0.24 \mu \mathrm{m}(n=16)$ in diameter immediately after sorting and exhibited typical stellate-shaped morphology, contained lipid vesicles with retinoids, and expressed eGFP as determined after excitation of living cells by blue and UV light after 2 days in culture (Figure 1C). Ectodermal and mesodermal stellate cell markers such as GFAP, nestin, vimentin, and desmin were detected by immunofluorescence in HSCs obtained by FACS (Figure 1, D-F), whereas hepatobiliary marker proteins such as keratins and $\alpha$-fetoprotein were undetectable (not shown). This indicated that typical HSCs were highly purified through the combination of density gradient centrifugation and retinoid-dependent FACS without contamination by other liver cells.

Transplantation of HSCs (2AAF/partial hepatectomy model). Freshly isolated HSCs from male $\mathrm{eGFP}^{+}$rats obtained by combined density gradient centrifugation and retinoid-dependent FACS were transplanted into female wild-type (WT) rats via tailvein injection immediately after liver injury through partial hepatectomy (PHX). The host animals were pretreated with 2AAF for 7 days in order to inhibit the proliferation of mature hepatocytes and to induce stem cell-based liver regeneration. We investigated 

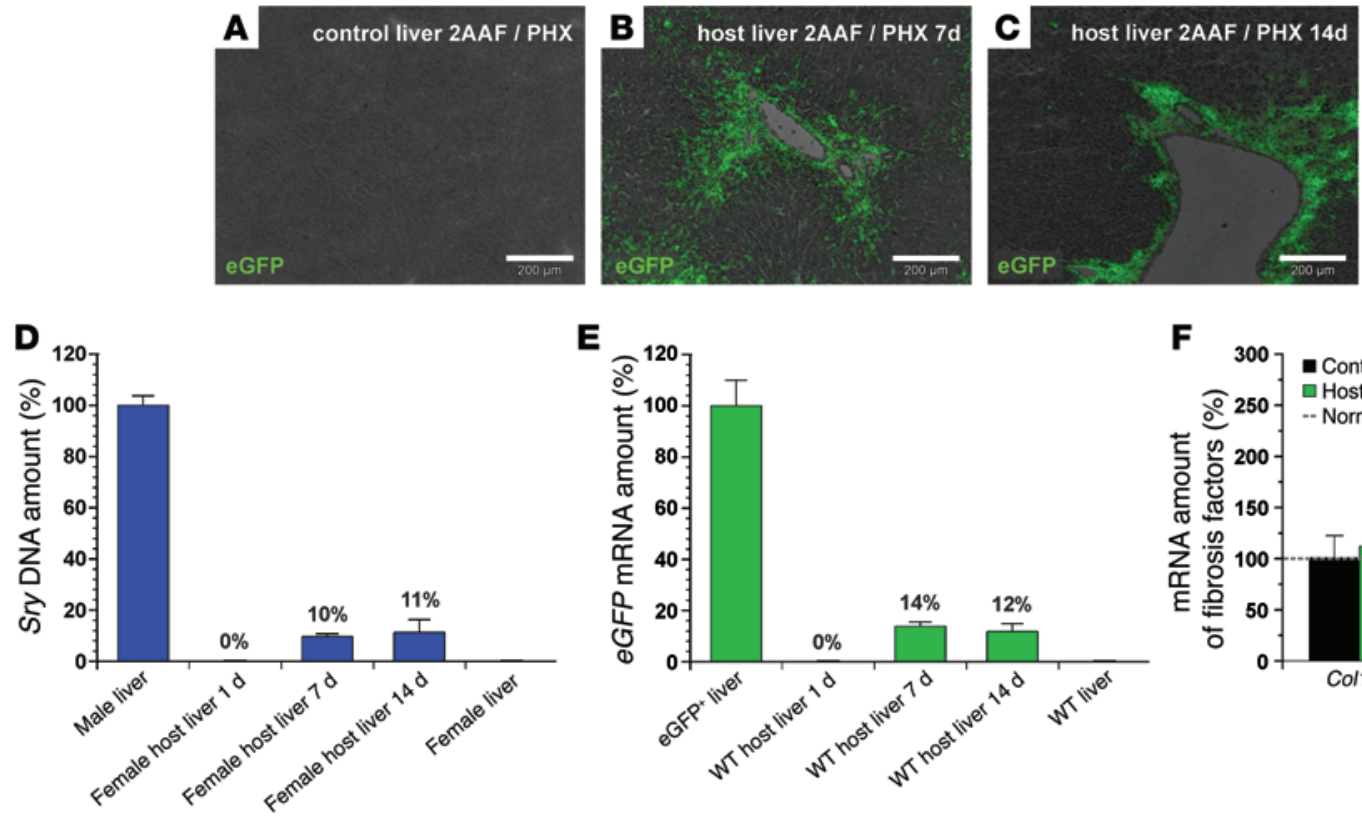

E

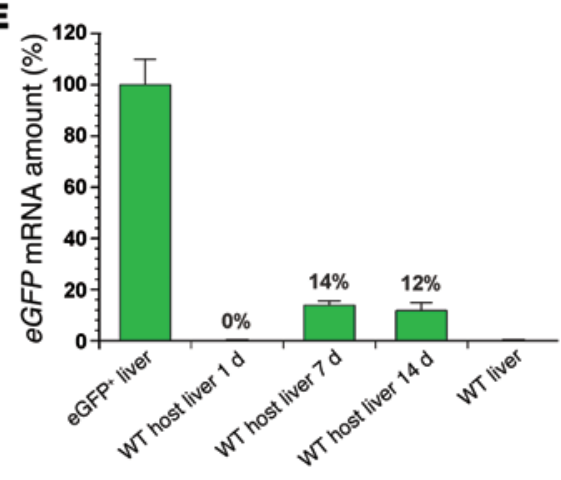

$\mathbf{F}$

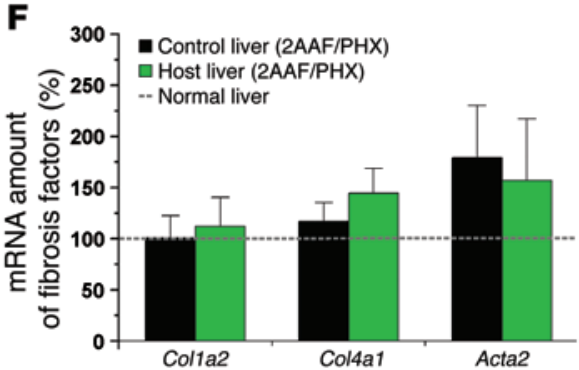

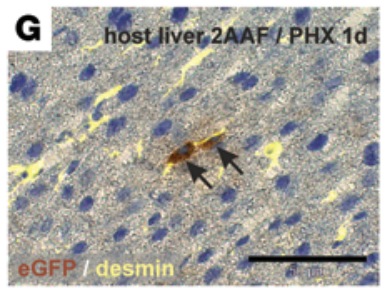
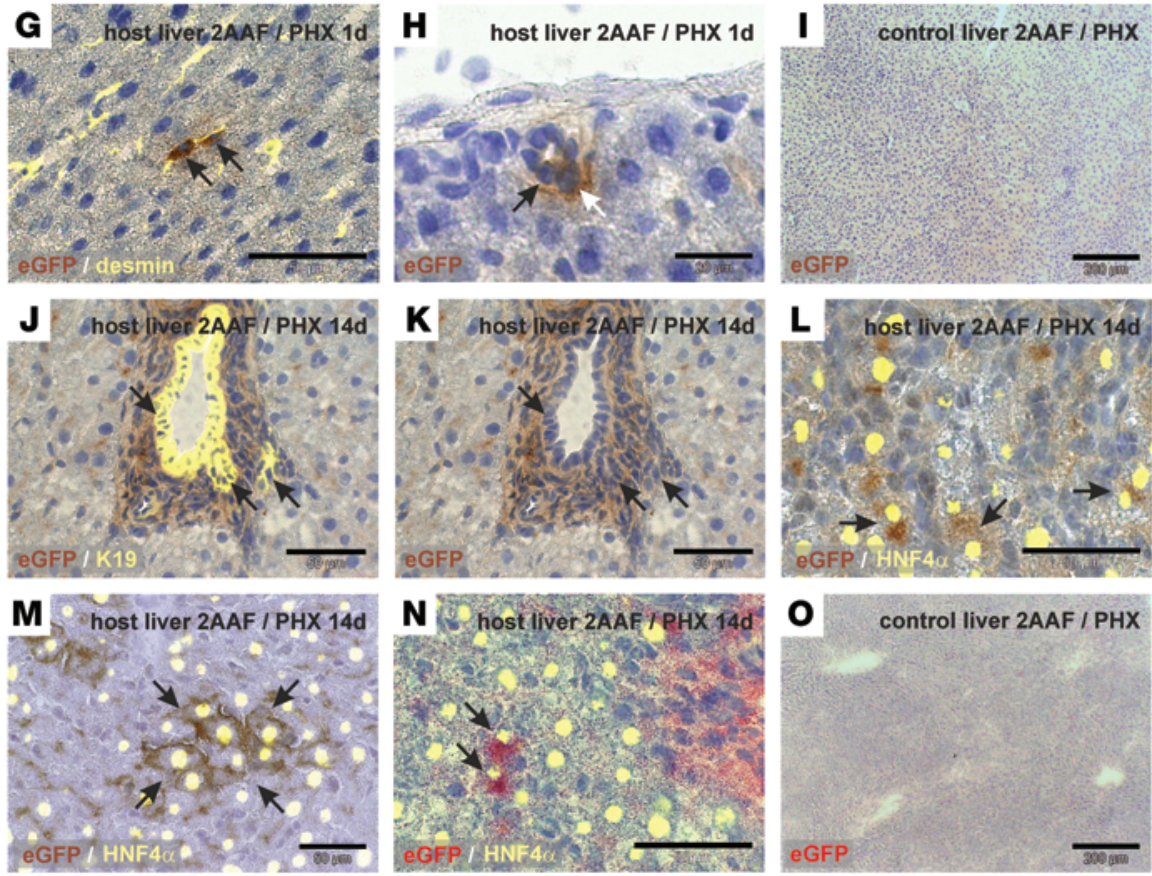

Figure 2. Transplanted eGFP+ HSCs home to the injured WT host liver and contribute to liver regeneration (2AAF/PHX model). eGFP immunofluorescence (green) of (A) WT control livers and WT host livers (B) 7 and (C) 14 days after HSC transplantation. qPCR of (D) Sry DNA and (E) eGFP mRNA on days 1,7 , and 14 after transplantation of male eGFP+ HSCs $(n=5)$. Livers of $(\mathbf{D})$ males $(n=6)$ and females $(n=4)$ or $(\mathbf{E})$ transgenic eGFP+ $(100 \%, n=3)$ and WT rats $(0 \%, n=3)$ were used for normalization. (F) qPCR of Col1a2 chain, Col4a1 chain, and Acta2 in rat livers from the 2AAF/PHX model with $(n=5)$ and without (control, $n=8$ ) transplanted HSCs on day 14. ( $\mathbf{G}$ and $\mathbf{H}$ ) DAB immunohistochemistry of eGFP (brown) in WT host liver 1 day after HSC transplantation. (G) eGFP+ HSCs were identified by desmin immunofluorescence (yellow; zone 2). (H) HSC-derived cells within (black arrow) or associated with (white arrow) bile ducts (zone 1). (I) WT control liver without DAB immunohistochemistry of eGFP. (J-M) DAB staining of eGFP (brown) 14 days after transplantation of eGFP+ HSCs. (J) K19-expressing cells (yellow) in bile ducts with (J and K) eGFP are indicated with arrows (zone 1). eGFP+ hepatocytes (brown; arrows) identified by HNF4 $\alpha$ immunofluorescence (yellow) (L) in zone 1 and (M) zone 3. (N) Fast red staining of eGFP' (red) hepatocytes with HNF4a (yellow; arrows; zone 1). (0) eGFP fast red staining of WT control liver. Scale bars: $200 \mu \mathrm{m}$ (A-C, I, and $\mathbf{0}$ ); $50 \mu \mathrm{m}$ (G and J-N); $20 \mu \mathrm{m}$ (H).

the localization of transplanted $\mathrm{eGFP}^{+} \mathrm{HSCs}$ in host livers by eGFP immunofluorescence after 7 and 14 days of liver regeneration. We found no significant eGFP staining in WT control livers (Figure 2A), whereas eGFP-expressing cells were detected mainly in the portal field of injured rat livers (2AAF/PHX model) that received $\mathrm{eGFP}^{+} \mathrm{HSC}$ (Figure 2, B and $\mathrm{C}$ ). We assessed the abundance of $\mathrm{eGFP}^{+}$male HSCs in female host livers by quantitative PCR (qPCR) analysis of male-specific sex-determining region $\mathrm{Y}$ 

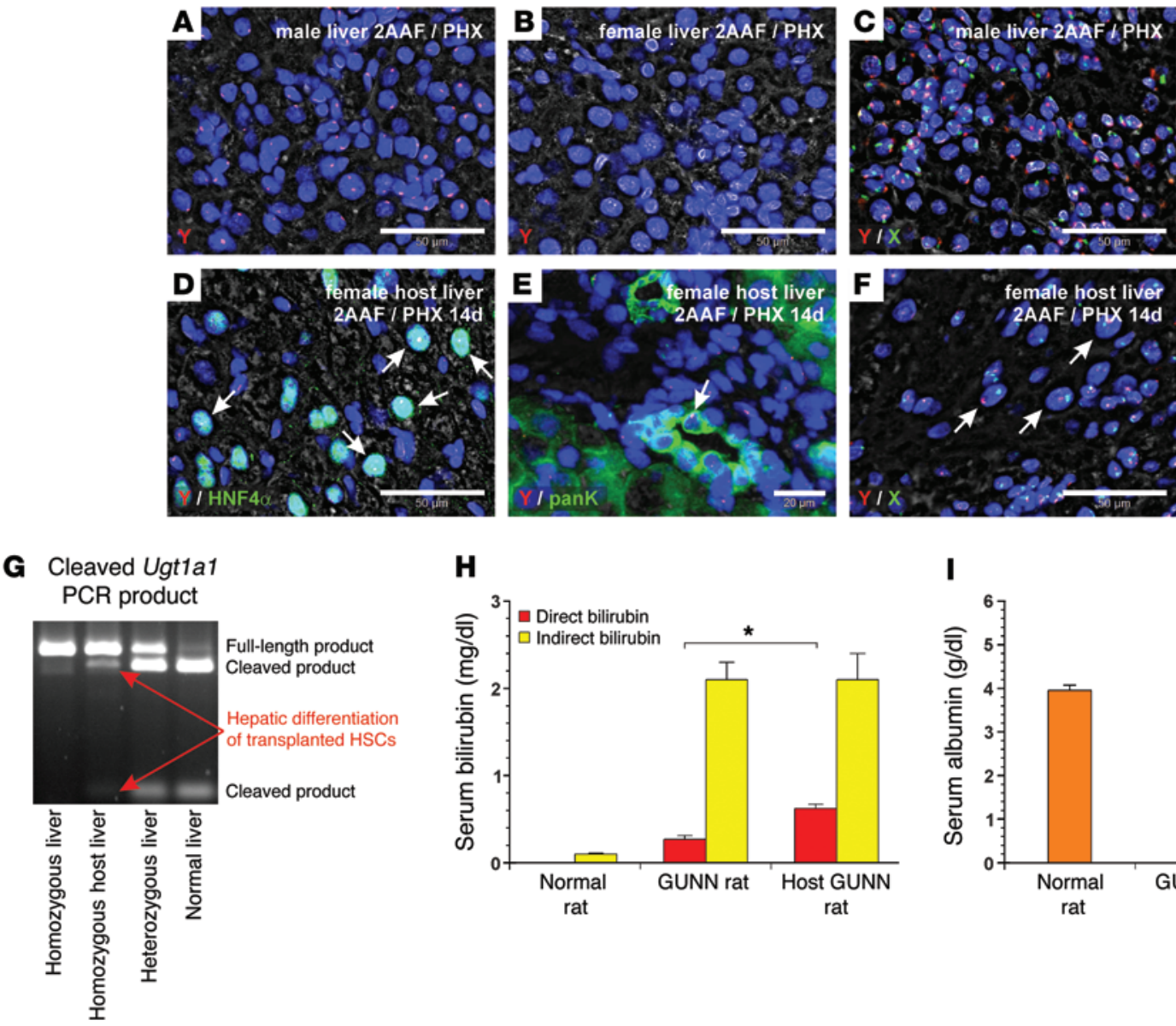

H

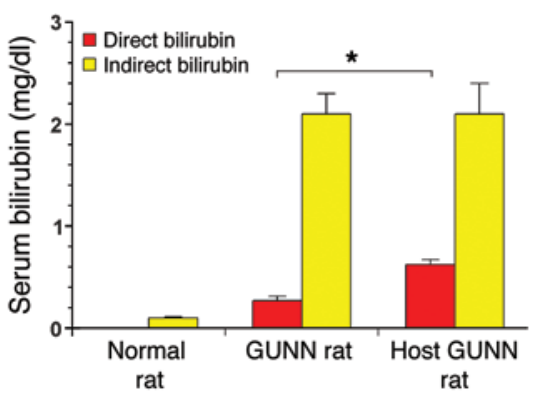

I

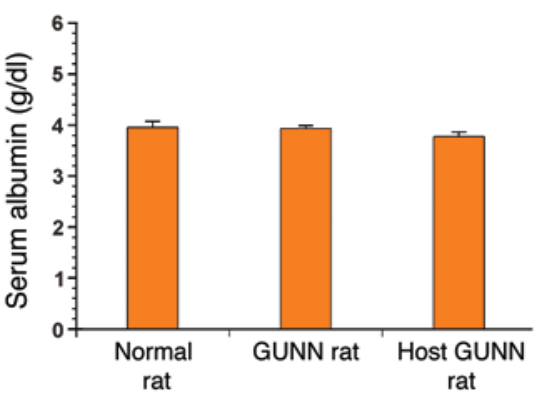

Figure 3. Transplanted male HSCs form bile duct cells and functional hepatocytes as investigated by FISH of gender-specific chromosomes and GUNN rats (2AAF/PHX model). (A) FISH of Y chromosome (red) on a male liver section was used as a positive control. (B) Female liver without positive FISH of Y chromosome. (C) FISH of Y (red) and X (green) chromosomes in male liver. Costaining of (D) HNF4 $\alpha$ (green) and (E) panK with FISH of Y chromosome (red) in female host livers after transplantation of male HSCs. Arrows indicate HSC-derived (D) hepatocytes and (E) bile duct cells with Y chromosome. (F) Detection of $\mathrm{Y}$ (red) and X (green) chromosomes in female host liver. Arrows indicate diploid and tetraploid HSC-derived male hepatocytes without cell fusion. Microscopic images were taken in the portal fields of injured livers (zone 1). (G-I) HSCs were transplanted into GUNN rats that underwent PHX in the presence of 2AAF. (G) The presence of hepatocytes derived from transplanted HSCs in homozygous GUNN rats was analyzed by RT-PCR of Ugt1a1 mRNA followed by enzymatic cleavage (BstNI) of the mutation site. (H) Serum concentrations of direct (red bars) and indirect bilirubin (yellow bars) were determined after 4 weeks and compared with those detected in GUNN rats (controls), which were treated in a similar way but without transplanted HSCs, and in normal rats $\left(n=3-6 ;{ }^{*} P<0.05\right)$. (I) Albumin concentrations were measured in the blood serum of normal rats as well as GUNN rats with and without transplanted HSCs $(n=3-6)$. Scale bars: $50 \mu \mathrm{m}$ (A-D and F); $20 \mu \mathrm{m}$ (E).

(Sry) DNA and eGFP mRNA (Figure 2, D and E). About $60 \%$ of the host animals that received eGFP ${ }^{+}$HSCs exhibited measurable Sry DNA or eGFP mRNA levels. Female host livers with stellate cell engraftment showed that approximately $10 \%$ to $14 \%$ of liver cells derived from transplanted male $\mathrm{eGFP}^{+} \mathrm{HSCs}$ after 7 and 14 days of regeneration, whereas HSC engraftment remained undetectable by qPCR in the host liver (Figure 2, D and E) and lung (not shown) 1 day after transplantation $(n=5)$. Conventional semiquantitative reverse transcriptase-polymerase chain reaction (RT-PCR) revealed the presence of $\mathrm{eGFP}^{+} \mathrm{HSCs}$ in host livers 1 day after transplantation (not shown). The eGFP mRNA levels within the WT host liver varied between $6 \%$ and 22\% (7-14 days) among different animals $(n=10)$ when qPCR was applied. The increase in Sry DNA and eGFP amounts during the first week indicated that transplanted HSCs proliferated in the host liver (Figure 2, D and E) without elevating the expression of fibrosis-associated markers such as collagen type $1 \alpha 2$ chain (Col1a2), Col4a1, or Acta 2 compared with the expression levels observed in injured control livers (2AAF/PHX model), as investigated by qPCR on days 1,7 , and 14 (shown for day 14 in Figure 2F). Expression of fibrosis-associated markers transiently increased in livers of the $2 \mathrm{AAF} / \mathrm{PHX}$ model during regeneration, with maximal expression around day 7 (not shown). We also observed engraftment of transplanted $\mathrm{eGFP}^{+}$ HSCs in the pancreas of WT host rats, as investigated by RT-PCR and qPCR of $e$ GFP mRNA. The $\mathrm{eGFP}^{+} \mathrm{HSCs}$ showed a low abundance of about $0.02 \%( \pm 0.01 \% ; n=4 ; 7$ days $)$ and $0.3 \%( \pm 0.09 \%$; $n=8 ; 14$ days) in the host pancreas. Pancreatic tissue from $\mathrm{eGFP}^{+}$ transgenic (100\%) and WT rats $(0 \%)$ were used for normalization.

Although transplanted $\mathrm{eGFP}^{+}$HSCs were below the detection limit of qPCR 1 day after transplantation (Figure 2, D and E), RT-PCR of eGFP and immunohistochemistry of eGFP using 3,3'-diaminobenzidine (DAB) revealed their presence in sinus- 

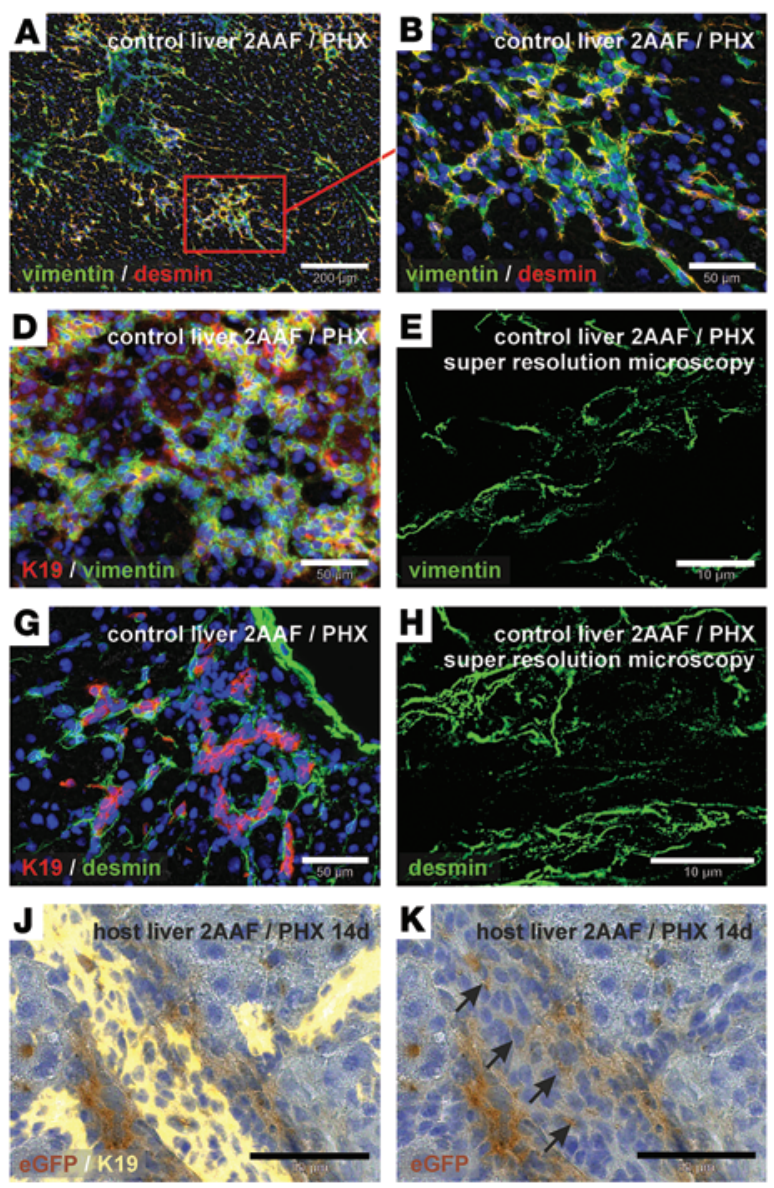
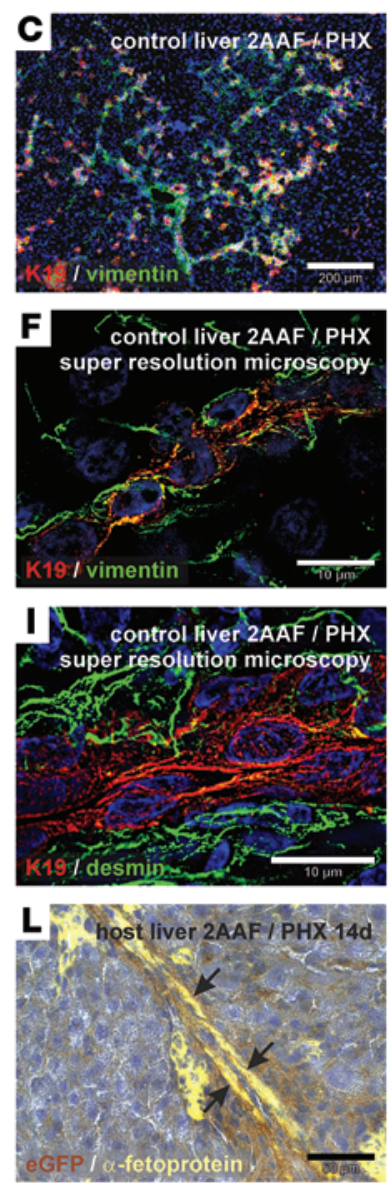

Figure 4. Transplanted eGFP+ ${ }^{+}$HSCs participate in ductular reactions (2AAF/PHX model). (A-I) Immunofluorescence of mesenchymal (vimentin and desmin) and epithelial (K19) marker proteins during ductular reactions in the liver after $\mathrm{PHX}$ in the presence of 2AAF. Simultaneous detection of vimentin (green) and desmin (red) in ductular structures of the portal field at (A) low and (B) high magnification. Combined immunofluorescence of K19 (red) and vimentin (green) at (C) low and (D) high magnification. Super-resolution microscopy of (E) vimentin (green) showing vimentin residues (F) in $\mathrm{K}^{1+}{ }^{+}$cells (red) in the center of duct-like structures. (G) Immunofluorescence of K19 (red) and desmin (green) of ductular structures within the portal field. (H and I) Desmin immunofluorescence (green) of ductular reactions was analyzed by super-resolution microscopy and $(\mathbf{H})$ showed desmin residues (I) in $\mathrm{K} 19^{+}$cells (red) within the center of duct-like structures. (J-L) DAB immunohistochemistry of eGFP (brown) in WT host liver with transplanted eGFP+ HSCs. (J) Epithelial cells of ductular reactions were indicated by (J) K19 and (L) $\alpha$-fetoprotein immunofluorescence (yellow). HSC-derived eGFP+ cells with (K) K19 (without immunofluorescence shown in J) or (L) $\alpha$-fetoprotein are indicated by arrows. All microscopic images were taken in the portal fields of injured livers (zone 1) Scale bars: $200 \mu \mathrm{m}$ (A and C); $50 \mu \mathrm{m}$ (B, D, G, and $\mathbf{J}-\mathbf{L}) ; 10 \mu \mathrm{m}(\mathbf{E}, \mathbf{F}, \mathbf{H}$, and $\mathbf{I})$. oids as desmin-expressing stellate cells and demonstrated their homing to the host liver (Figure $2 \mathrm{G}$ ). We also found eGFP ${ }^{+} \mathrm{HSCs}$ to be integrated and associated with bile ducts in the host liver 1 day after transplantation (Figure $2 \mathrm{H}$ ). WT control livers (2AAF/ PHX model) displayed no DAB staining of eGFP (Figure 2I). DAB immunohistochemistry of eGFP, in combination with immunofluorescence of the bile duct marker K19 and hepatocyte marker HNF $4 \alpha$, indicated that transplanted HSCs contributed to bile duct and hepatocyte formation after 14 days of liver regeneration (Figure 2, J-M). We found HSC-derived hepatocytes in both the periportal (zone 1; Figure 2L) and pericentral (zone 3; Figure $2 \mathrm{M})$ zones. The differentiation of HSCs into hepatocytes was also investigated by fast red immunohistochemistry of eGFP and immunofluorescence of $\mathrm{HNF} 4 \alpha$ (Figure 2N). We did not observe positive fast red immunohistochemistry of eGFP in WT control livers (2AAF/PHX model) (Figure 2O). We found a similar differentiation potential within the regenerating liver (2AAF/PHX) when HSC clones, which derived from single eGFP ${ }^{+}$HSCs by clonal expansion, were transplanted. As indicated by qPCR analysis of $e$ GFP mRNA, different HSC clones reconstituted about $16 \%$ ( $\pm 5 \%$; range $3 \%-28 \% ; n=4$ ) of the host liver (Supplemental Figure $1, \mathrm{~A}-\mathrm{C}$ ). We investigated the differentiation of male HSC clones into epithelial cells within the female host liver by fluorescence in situ hybridization (FISH) of male-specific $\mathrm{Y}$ chromosome. FISH analysis revealed HSC-derived Y chromosome in hepatocytes and bile duct cells when immunofluorescence of $\mathrm{HNF} 4 \alpha$ or keratins (pan keratin, panK) was added (Supplemental Figure 1, B and $\mathrm{C}$ ). We verified the specificity of the $\mathrm{Y}$ chromosome probe on male (positive control) and female (negative control) liver sections (Figure 3, A and B) and by combining $\mathrm{Y}$ and $\mathrm{X}$ chromosome probes through distinct signals for each chromosome in the cell nuclei of male control liver sections (Figure 3C). After transplantation of primary HSCs from male rats sorted by FACS, Y chromosome was detected by FISH in $\mathrm{HNF}_{4} \alpha^{+}$and panK ${ }^{+}$cells within the female host liver (2AAF/PHX model), indicating HSC-derived hepatocytes and bile duct cells within the female host liver (Figure 3, D and E). Simultaneous FISH of Y and X chromosomes displayed normal diploid and tetraploid hepatocytes that derived from transplanted male HSCs without fusion with preexisting female hepatocytes (Figure 3F). Transplanted HSCs were able to restore liver function of homozygous GUNN rats, which lack uridine 5'-diphospho-glucuronosyltransferase 1A1 (UGT1A1) activity due to mutation in exon 4. The UGT1A1 enzyme is expressed in hepatocytes and required for bilirubin glucuronidation (conjugation), which enables bilirubin excretion. Owing to Ugt1a1 deficiency, blood sera from homozygous GUNN rats exhibit high bilirubin serum levels compared with levels in normal rats. We verified the presence of Ugt1a1 transcripts without mutation in homozygous GUNN rat livers after transplantation of HSCs by enzymatic cleavage (BstNI) of Ugt1a1 PCR products, which results in additional PCR products similar to those obtained in heterozygous GUNN or normal rats. In contrast, mutated Ugt1a1 remained uncleaved and appeared as 

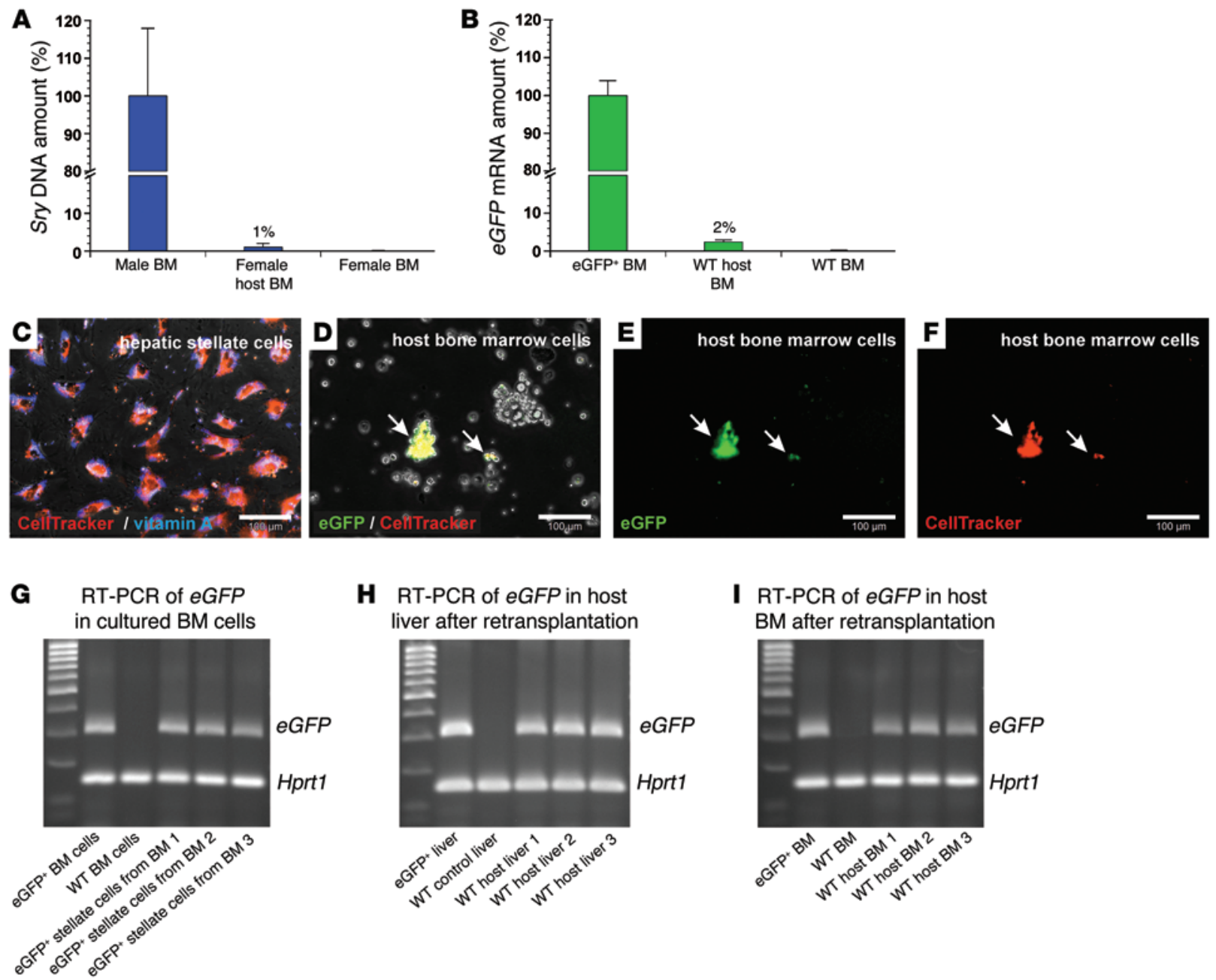

H RT-PCR of eGFP in host liver after retransplantation
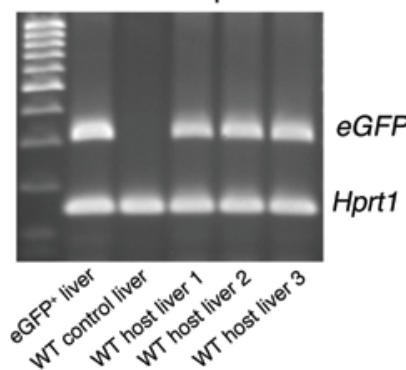

I RT-PCR of eGFP in host BM after retransplantation

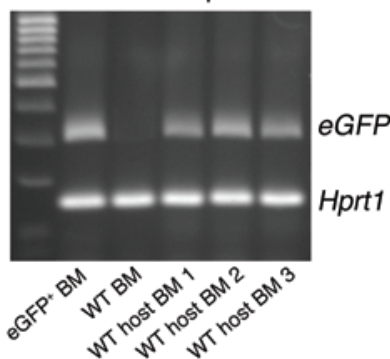

Figure 5. Engraftment of transplanted eGFP+ HSCs in the BM of WT host animals and their retransplantation (2AAF/PHX model). qPCR of (A) Sry DNA and (B) eGFP mRNA within 14 days after HSC transplantation $(n=5)$. The relative Sry DNA or mRNA amount of BM samples from male or eGFP+ rats (100\%, $n=4)$ and female or WT rats $(0 \%, n=4)$ were used for normalization. (C) Fluorescent CellTracker (red) staining of isolated HSCs. HSC identity was verified by vitamin A fluorescence (blue). (D-F) eGFP+ HSCs stained with CellTracker were injected into WT rats (2AAF/PHX model) and isolated from the BM 1 day after transplantation. (D) Transmission light microscopy and merged fluorescence of (E) eGFP and (F) CellTracker (red) staining of BM cultures. Arrows indicate $\mathrm{eGFP}^{+}$and CellTracker+ HSC-derived cells. (G) BM cells with eGFP+ ${ }^{+} \mathrm{HSC}$-derived cells were expanded in culture, and the presence of eGFP mRNA was verified by RT-PCR after 14 days $(n=3)$. BM of eGFP+ and WT rats served as positive and negative controls for eGFP expression. (H) eGFP+ HSC-derived cells in BM cell cultures (shown in $\mathbf{G}$ ) were retransplanted into 3 different WT rats (2AAF/PHX model) and detected in the (H) liver and (I) BM of all host animals after 14 days by RT-PCR of eGFP mRNA. Hprt1 mRNA was used to demonstrate equal mRNA amounts in all samples. Scale bars: $100 \mu \mathrm{m}$ (C-F).

a single PCR product at $465 \mathrm{bp}$ (Figure 3G). In line with this, serum levels of conjugated (direct) bilirubin in homozygous GUNN rats increased 2-fold within 4 weeks after transplantation of HSCs derived from rats with normal Ugt1a1 when compared with serum levels in GUNN rats without transplanted HSCs (Figure 3H). This indicates partial correction of the bilirubin-handling defect in GUNN rats through differentiation of transplanted HSCs into hepatocytes, although the amounts of unconjugated (indirect) bilirubin were not yet significantly altered (Figure $3 \mathrm{H}$ ). The albumin serum concentrations remained constant (Figure 3I).

Transplanted HSCs and ductular reactions (2AAF/PHX model). The ductular reaction is regarded as a response of stem/progenitor cells to severe liver injury and is formed by $\mathrm{K} 19^{+}$progenitor cells as well as surrounding mesenchymal cells. These ducts transiently appeared in the $2 \mathrm{AAF} / \mathrm{PHX}$ model and were found in the regenerating liver from day 7 to day 14 after PHX (Figure 4). Mesenchymal marker proteins such as vimentin and desmin were highly expressed in liver tissue with ductular reactions (Figure 4, A and B). Progenitor cells that express the mesodermal protein vimentin and the epithelial marker K19 constituted the core of the ducts (Figure 4, C-F) surrounded by cells that expressed the stellate cell markers desmin (Figure 4G) and nestin (not shown). We verified the presence of vimentin in $\mathrm{K} 19^{+}$cells within these ductlike structures by immunofluorescence and ELYRA super-resolution microscopy (Zeiss) (Figure 4, E and F). Although desmin- and K19-expressing cells seem to be clearly distinct in these structures (Figure 4G), super-resolution microscopy revealed the presence of desmin residues in $\mathrm{K} 19^{+}$cells (Figure $4, \mathrm{H}$ and I), indicating that intermediate states of mesenchymal and epithelial cells exist in putative progenitor cells of the liver. We found transplanted eGFP ${ }^{+}$ 

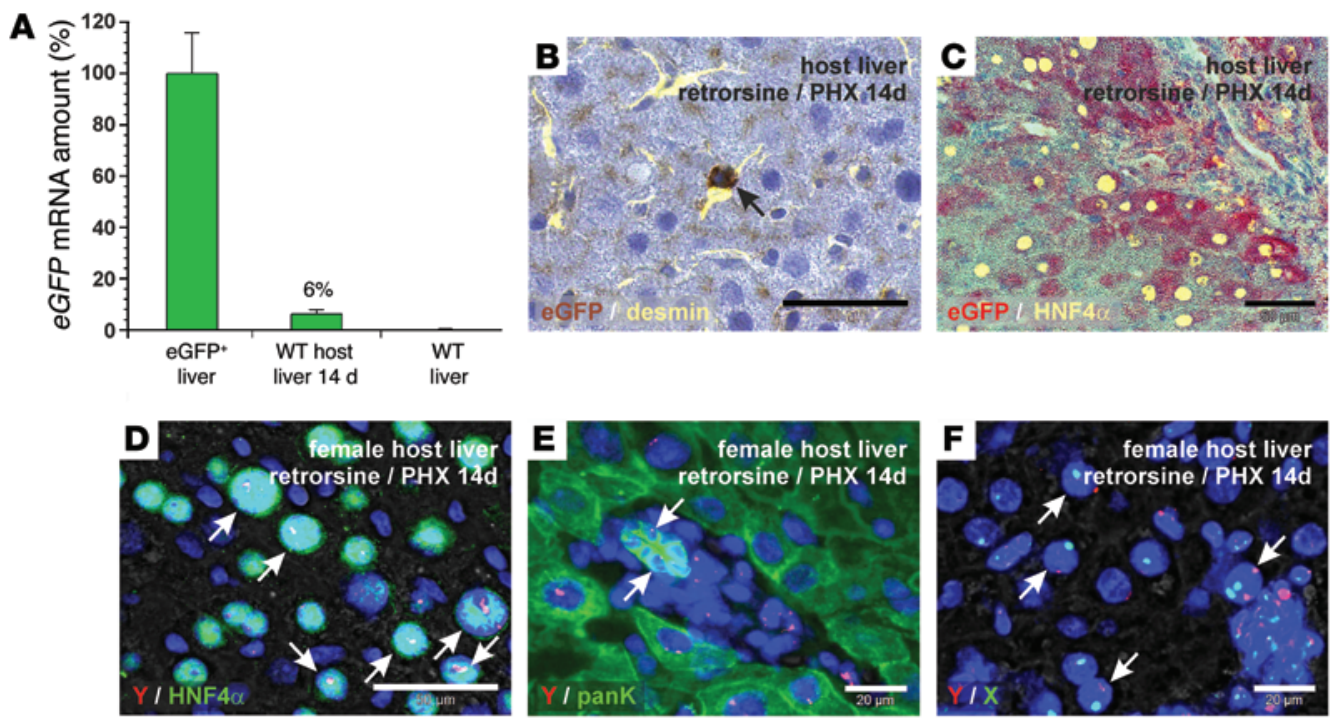

Figure 6. Transplanted eGFP+ HSCs contribute to liver regeneration in WT host rats that undergo PHX in the presence of retrorsine. (A) Percentage of cells that derived from transplanted eGFP+ male HSCs in female host liver within 14 days of liver regeneration was assessed by qPCR of $e G F P$ mRNA ( $n=7$ ). Livers of transgenic eGFP' $(100 \%, n=3)$ and WT rats $(0 \%, n=3)$ were used for normalization. (B) DAB immunohistochemistry of eGFP (brown) and desmin immunofluorescence (yellow) in WT host liver (zone 2). (C) Fast red immunohistochemistry of eGFP (red) in combination with HNF4 $\alpha$ immunofluorescence (yellow) was used to identify hepatocytes derived from transplanted eGFP+ HSCs. Combined FISH of Y chromosome (red) and (D) immunofluorescence of HNF4 $\alpha$ or (E) panK (green) in female host liver (zone 1). (D) Hepatocytes and (E) bile duct cells derived from transplanted male HSCs are indicated by arrows. (D) The $Y$ chromosome (red) appeared white when HNF4 $\alpha$ (green) and DAPI (blue) were costained. (F) Simultaneous detection of $Y$ (red) and $X$ (green) chromosomes by FISH in female host liver. Arrows indicate diploid and tetraploid hepatocytes that derived from transplanted male HSCs without cell fusion (zone 1). Scale bars: $50 \mu \mathrm{m}$ (B, C, and D); $20 \mu \mathrm{m}$ (E and F).

HSCs in the host liver in areas with ductular reactions by day 7 of liver regeneration (2AAF/PHX model), as shown by DAB immunohistochemistry of eGFP. Simultaneous immunohistochemical staining of eGFP with the progenitor cell markers K19 and $\alpha$-fetoprotein indicated that transplanted $\mathrm{eGFP}^{+} \mathrm{HSC}$ contributed to putative epithelial progenitors that formed duct-like structures (Figure 4, J-L). Although we found HSC-derived $\mathrm{eGFP}^{+}$and $\mathrm{K} 9^{+}$ cells among ductular cells, the majority of $\mathrm{eGFP}^{+}$cells seemed to surround the ducts as mesenchymal tissue (Figure 4, J and $\mathrm{K}$ ).

Retransplantation of HSCs (2AAF/PHX model). In addition to their presence in the liver, we also found engraftment of transplanted HSCs (1\%-2\%; $n=5$; Figure 5 , A and B) and HSC clones $(1 \% ; n=5$; not shown) in the $\mathrm{BM}$ of host rats as investigated by qPCR of Sry DNA and eGFP mRNA 7-14 days after tail-vein injection. Since cells of the BM can be gently isolated from long bones without enzymes, we used this source for retransplantation experiments with stellate cells. FACS-sorted eGFP ${ }^{+}$HSCs were first stained by CellTracker Red and then transplanted into WT rats immediately after PHX in the presence of 2AAF. One day after transplantation, the host $\mathrm{BM}$ cells were harvested and cultured. The intense CellTracker fluorescence, as demonstrated for freshly isolated HSCs (Figure 5C), was used to find transplanted HSCderived $\mathrm{eGFP}^{+}$cells among WT BM cells (Figure 5, D-F), which finally converted into myofibroblast-like cells during culture (not shown). eGFP expression in HSC-derived cells was maintained during culture (Figure $5 \mathrm{G}$ ) and used to analyze their engraftment in the liver and BM 14 days after their transplantation into new host rats (2AAF/PHX model) (Figure 5, H and I). The retransplanted eGFP ${ }^{+} \mathrm{HSC}$-derived cells were able to reconstitute about
$17 \%( \pm 5 \% ; n=3)$ of the injured host livers as investigated by qPCR of $e G F P$ mRNA. The percentage of eGFP' cells varied in the different areas of the host livers (Supplemental Table 1).

Transplantation of HSCs (retrorsine/PHX model). FACS-sorted HSCs from $\mathrm{eGFP}^{+}$male rats were also injected into WT female rats that underwent PHX in the presence of retrorsine, which prevents the proliferation of mature hepatocytes to facilitate engraftment of transplanted cells in the injured liver. In this model, transplanted eGFP ${ }^{+}$HSCs formed about $6 \%( \pm 2 \%$; range $1 \%-14 \%$; $n=7$ ) of the host liver, as investigated by qPCR of $e G F P$ mRNA (Figure 6A). Engraftment of transplanted eGFP ${ }^{+} \mathrm{HSC}$ s was detectable by QPCR in all host rats when the retrorsine/PHX model was applied. The $\mathrm{eGFP}^{+} \mathrm{HSC}$ s were maintained as stellate cells in the liver sinusoids of the host liver 14 days after their transplantation (retrorsine/PHX model), as demonstrated by combined DAB immunohistochemistry of eGFP and immunofluorescence of desmin (Figure 6B). Immunohistochemistry of eGFP (fast red) and immunofluorescence of the hepatocyte marker HNF4 $\alpha$ revealed that transplanted $\mathrm{eGFP}^{+} \mathrm{HSCs}$ also differentiated into hepatocytes (Figure 6C). The generation of hepatocytes by male HSCs in female host liver was confirmed by FISH of Y chromosome in conjunction with HNF4 $\alpha$ immunofluorescence (Figure 6D). We also found the Y chromosome in bile duct cells when Y chromosome probes were combined with panK immunofluorescence (Figure $6 \mathrm{E})$. We found that the transplanted male HSCs were able to generate hepatocytes in the female host liver without cell fusion, as determined by FISH of $\mathrm{Y}$ and $\mathrm{X}$ chromosomes (Figure 6F).

Hepatic differentiation of MSC populations in vitro. Participation of transplanted HSCs in ductular reactions and the potential of 
A

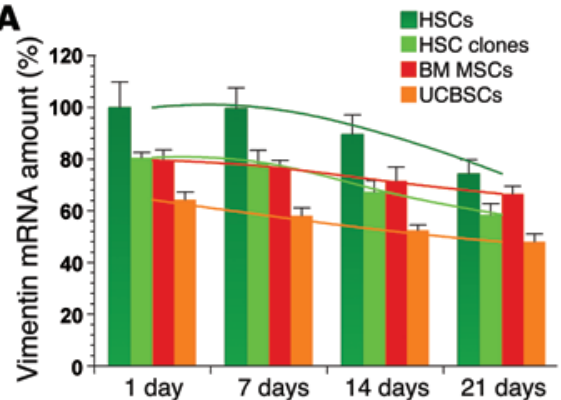

D

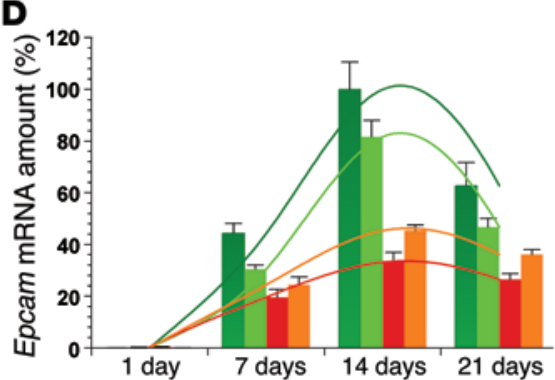

G

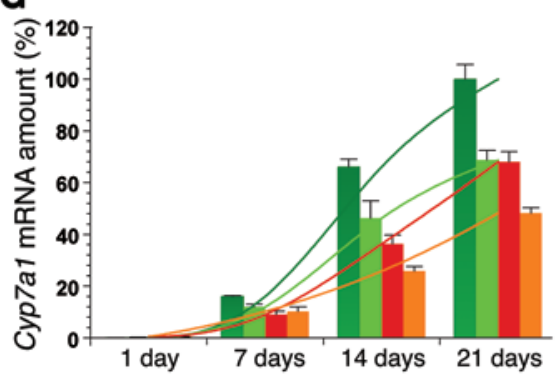

J
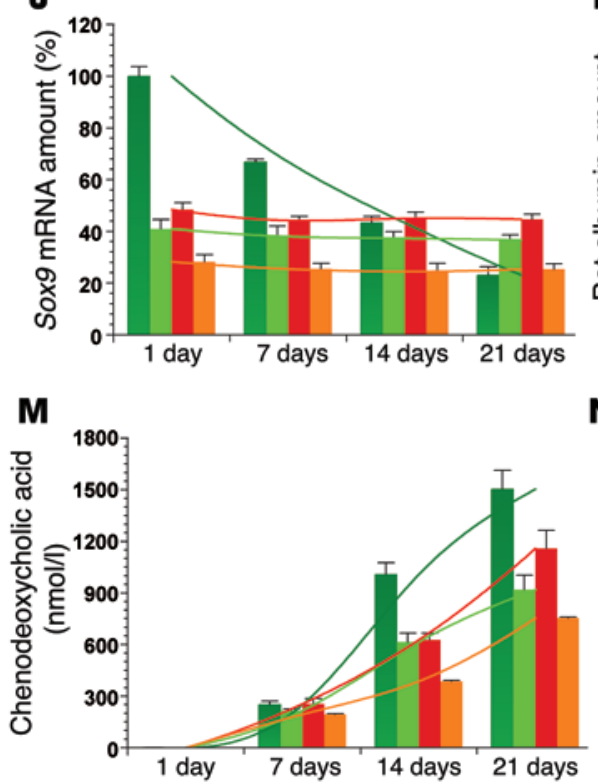

B

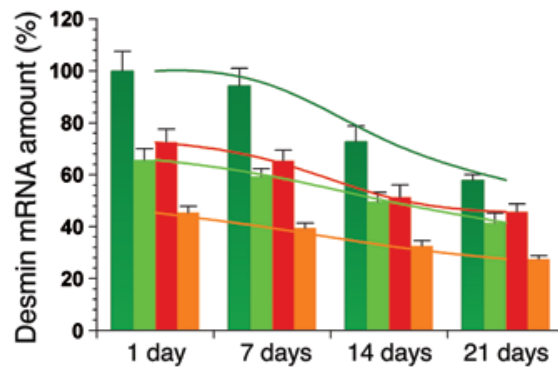

E

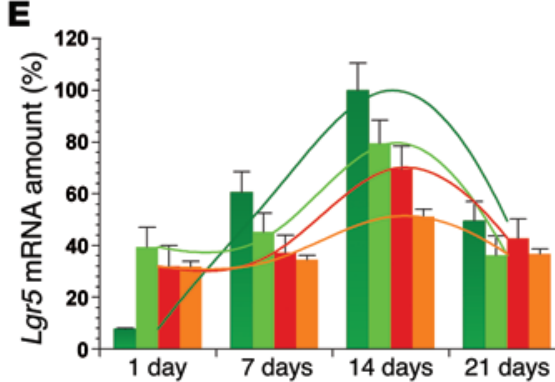

H

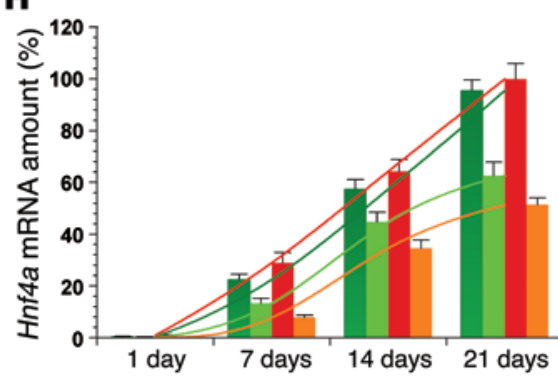

K

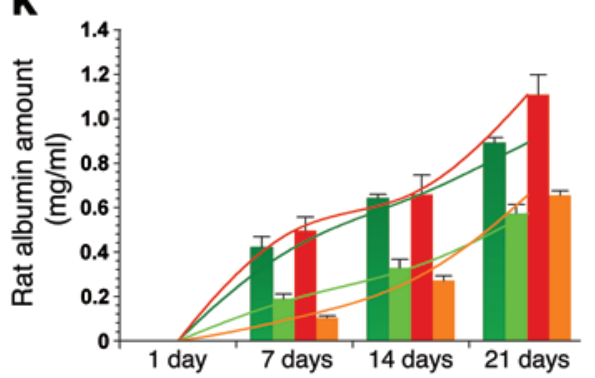

N

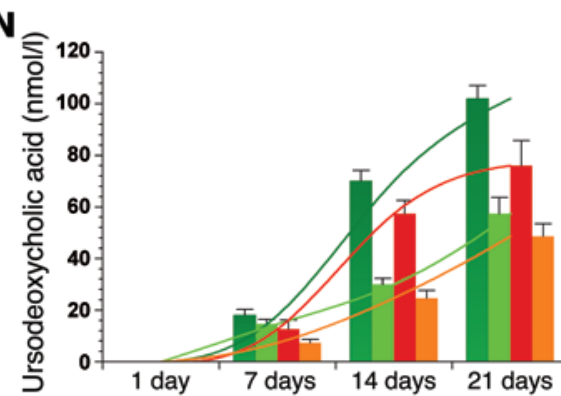

C

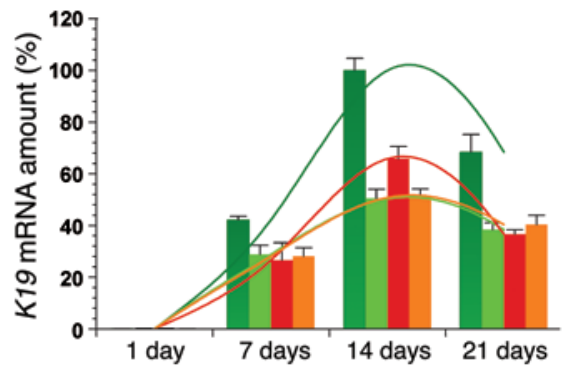

F
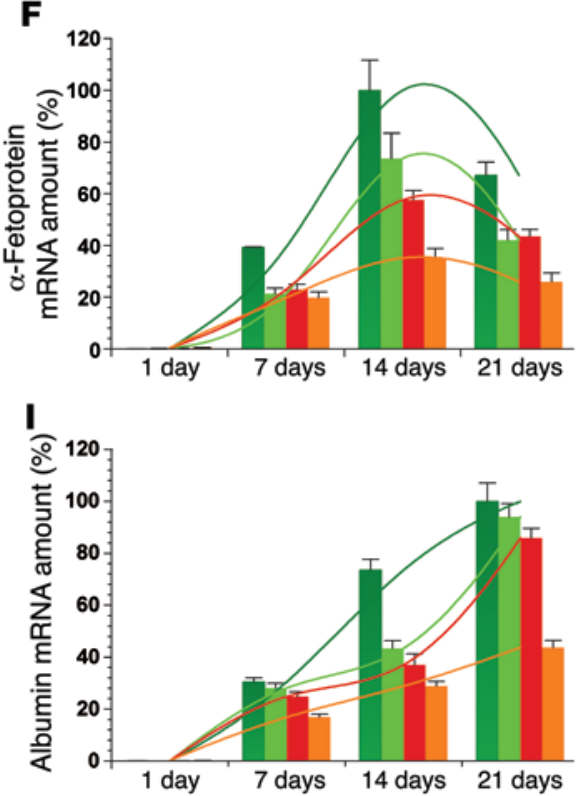

$\mathbf{L}$
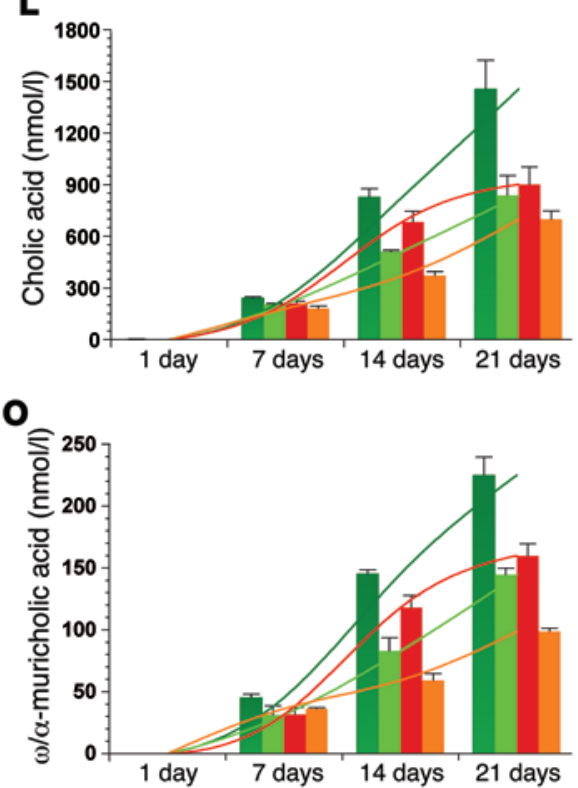

Figure 7. MSCs transiently acquire the gene expression of liver progenitor cells during their differentiation into hepatocytes. (A-0) Freshly isolated HSCs, clonally expanded HSCs (clones 1E7, 4E7, and 3F8), BM MSCs, and UCBSCs (clone 1C11) from rats were treated for 21 days with a hepatocyte differentiation medium that contained HGF and FGF4. Expression of (A) vimentin, (B) desmin, (C) K19, (D) Epcam, (E) Lgr5, (F) $\alpha$-fetoprotein, (G) Cyp7a1, (H) Hnf4a, (I) albumin, and (J) Sox 9 was analyzed by qPCR. mRNA samples were taken at weekly intervals. Gene expression of the cells at the beginning of the differentiation experiments is indicated as Day 1. (K) To verify hepatic differentiation, the hepatocyte marker albumin was also measured in the culture supernatant of HSCs, HSC clones, BM MSCs, and UCBSCs by a rat-specific albumin ELISA. Hepatic function of MSC-derived hepatocyte-like cells was also determined by quantitation of the bile acids (L) cholic acid, (M) chenodeoxycholic acid, (N) ursodeoxycholic acid, and (0) $\omega / \alpha$-muricholic acid in culture supernatants by UHPLC-MS/MS. Bile acids were allowed to accumulate for 2 days in the culture medium before analysis. Bar values represent arithmetic means and were interpolated to indicate the trend (colored lines) for each cell group. Expression profiles, albumin synthesis, and bile acid release were determined in 3 independent experiments $(n=3$ ). 
HSCs to develop into hepatocytes (Figure 2, M and N, and Figure 3D) and cholangiocytes (Figure 2, K and L, and Figure 3E) raise the possibility that stellate cells could transiently acquire the expression pattern of liver progenitor cells during hepatic differentiation. Stellate cells were recently described as liver-resident MSCs (8) and showed an expression profile similar to that of clonally expanded HSCs, rat BM MSCs, and umbilical cord blood stem cells (UCBSCs) (Supplemental Figure 2). We treated these MSC populations with hepatocyte growth factor (HGF) and human fibroblast growth factor 4 (FGF4) for 21 days in order to monitor hepatic differentiation. The relative mRNA amounts of stellate cell or mesenchymal markers (vimentin, desmin, Lrat), indicators for progenitor cells and putative hepatic stem/progenitor cells (K19, Epcam, leucine-rich repeat-containing $\mathrm{G}$ protein-coupled receptor 5 [Lgr5], $\alpha$-fetoprotein) as well as markers of mature hepatocytes (cytochrome P450 7A1 [Cyp7a1], Hnf4a, albumin) were analyzed by qPCR (Figure 7 and Supplemental Figure 3, A-C). The expression levels of vimentin, desmin, and Lrat steadily decreased by $20 \%$ to $30 \%$ during hepatic differentiation (Figure 7, A and B, and Supplemental Figure 3, A and B), indicating that only a subset of MSCs had differentiated in vitro. In contrast, the expression levels of K19, Epcam, Lgr5, and $\alpha$-fetoprotein transiently increased and peaked on day 14 during hepatic differentiation of cultured HSCs and other MSC populations (Figure 7, C-F). The mRNA values of the hepatocyte markers Cyp7a1, $H n f 4 a$, and albumin continuously increased until day 21 of differentiation in all MSCs (Figure 7, G-I), indicating final differentiation into the hepatic cell lineage. The mRNA values of sex-determining region Y box 9 (Sox9) decreased in primary cultured HSCs in both the growth factor-treated (Figure 7J) and control groups (Supplemental Figure 3C), whereas the Sox9 levels of HSC clones and other MSC populations remained unchanged in all experimental media (Figure 7J). This may indicate that Sox 9 was apparently not involved in hepatic differentiation. Neither progenitor cells (K19, Epcam, or $\alpha$-fetoprotein) nor hepatocyte markers (Cyp7a1, Hnf4a, or albumin) were detected by qPCR in the controls without growth factors over a 21-day culture, as demonstrated for albumin expression in Supplemental Figure 3D. We confirmed the appearance of hepatocyte-like cells by quantification of albumin in the culture supernatant of the MSCs using a rat-specific albumin enzyme-linked immunosorbent assay (ELISA) (Figure 7K). In contrast to HSCs, muscle fibroblasts were unable to respond by albumin mRNA expression or protein synthesis after growth factor treatment for 21 days (Supplemental Figure 3, D and E). Also, the release of bile acids such as cholic acid, chenodeoxycholic acid, ursodeoxycholic acid, and $\alpha / \omega$-muricholic acid into the culture supernatants indicated that all MSC populations acquired hepatocyte-specific functions during growth factor treatment (Figure 7, L-O). In the culture media of HGF- and FGF4treated MSCs, 19 of 29 investigated bile acids were detected by ultra-high-performance liquid chromatography-tandem mass spectrometry (UHPLC-MS/MS) (Supplemental Figures 4-7), whereas bile acid synthesis was not found under control conditions without cytokines within a 21-day period (Supplemental Figure 3F and Supplemental Tables 2-5). With the exception of Sox9 expression in HSC clones - BM MSCs and UCBSCs that remained unchanged (Figure 7J) - all trends in gene expression, albumin synthesis, and bile acid synthesis were significant, as determined by ANOVA (not indicated in Figure 7; see Supplemental Figure 3).
We also investigated the transient appearance of putative progenitor cells in differentiation experiments of primary cultured HSCs using combined immunofluorescence of desmin with K19, $\alpha$-fetoprotein, or LGR5. Small cells with K19 filaments, $\alpha$-fetoprotein, or LGR5 exhibited desmin filaments 14 days after HGF and FGF4 treatment (Figure 8, A-C). Only LGR5 was detectable under control conditions in myofibroblast-like cells, which typically develop from culture-activated stellate cells, whereas K19 and $\alpha$-fetoprotein remained undetectable (Figure 8, D-F). Hepatocyte-like cells with residual desmin filaments in HSC primary cultures were identified by immunofluorescence of bile salt export pump (BSEP), sodium taurocholate cotransporting polypeptide (NTCP), and K18 21 days after we initiated the growth factor treatment (Figure 8, G-L). In control experiments without HGF and FGF4, HSC-derived myofibroblasts expressed desmin but remained negative for BSEP, NTCP, and K18 within 21 days of culture (Figure 8, M-O). In summary, we found that stellate cells and other MSCs reduced their mesodermal characteristics during differentiation into hepatocytes (endoderm), which was accompanied by a transient appearance of progenitor cell markers (Supplemental Figure 8).

\section{Discussion}

This study demonstrates for the first time to our knowledge that HSCs are transplantable, home to the liver of host animals, and participate in liver regeneration through differentiation into parenchymal and bile duct cells without cell fusion. Transplanted HSCs were able to ameliorate the glucuronidation defect in GUNN rats, as indicated by increased serum levels of conjugated bilirubin and the appearance of nonmutated UGT1A1 enzymes in the liver of these animals. This confirms the contribution of stellate cells to the formation of functional hepatocytes, although total bilirubin serum concentrations in host GUNN rats were not significantly lowered. A complete resolution of the bilirubin-handling defect may require higher numbers of transplanted HSCs and additional time for bilirubin clearance. In addition to these findings obtained with freshly isolated HSCs, single-cell clones of HSCs were established that preserved stellate cell characteristics and the ability to differentiate, indicating a self-renewal potential of HSCs. These important stem cell features further support the characterization of stellate cells as liver-resident MSCs $(8,16)$. In line with their characterization as MSCs, engraftment of transplanted HSCs was also found in the BM of host animals, as demonstrated in the present study. MSCs from BM or adipose tissue were reported to contribute to liver repair (25-27), which suggests that hepatic differentiation capacity is a general feature of MSCs. There is currently no consensus about a direct contribution of stem cells with mesodermal characteristics to epithelial tissue regeneration in the liver, although mesodermal proteins such as vimentin are expressed by progenitor cells along with epithelial markers (35). It is assumed that stem cells with epithelial characteristics are the source of liver progenitor cells (34), which ensure tissue repair in the event of impaired hepatocyte proliferation $(32,33)$. In line with this, epithelial markers such as K19 and EPCAM are expressed in ductular reactions (35), which are described as a stem/progenitor cell response (36). However, the simultaneous appearance of epithelial and mesodermal proteins in liver progenitor cells has remained 

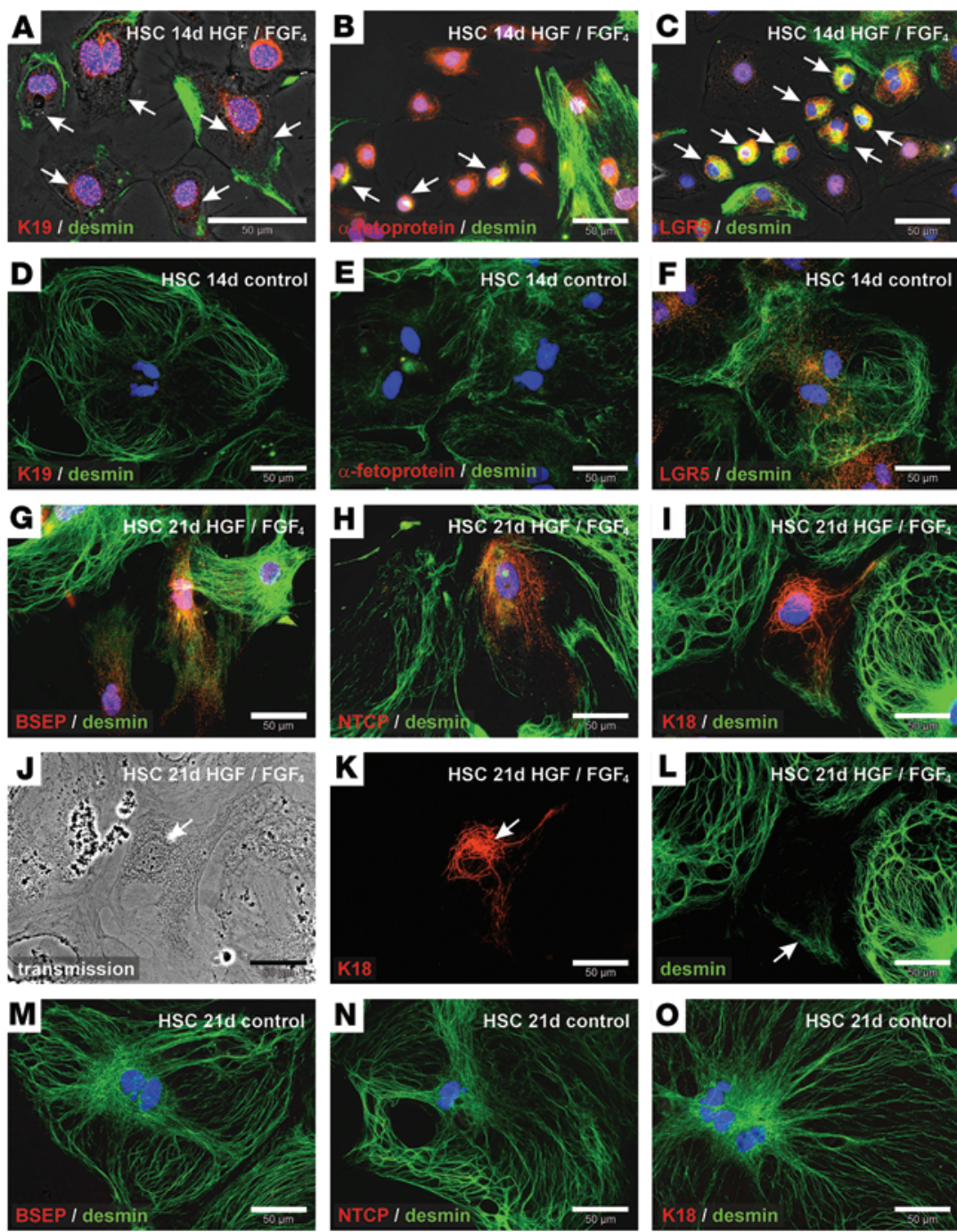
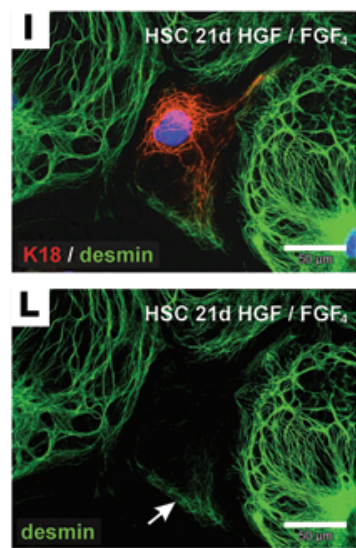

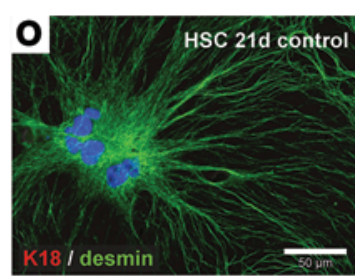

Figure 8. Isolated HSCs form intermediate states of mesenchymal and epithelial cells during differentiation into hepatocyte-like cells. Combined immunofluorescence of (A) K19 and (B) $\alpha$-fetoprotein as well as (C) LCR5 (red) with the mesodermal filament protein desmin (green) after treatment of primary HSC cultures with HGF and FGF4 for 14 days. Small cells with K19, $\alpha$-fetoprotein, or LCR5 that coexpressed desmin are indicated with arrows. HSCs without growth factors (control) were also analyzed by immunofluorescence of (D) K19, (E) $\alpha$-fetoprotein, or (F) LGR5 (red) in combination with desmin (green) after 14 days of culture. Hepatic differentiation of HSCs was analyzed by immunofluorescence of (G) BSEP, (H) NTCP, and (I) K18 (red) after 21 days of growth factor treatment. (G-L) To investigate the relationship of newly formed hepatocyte-like cells with HSCs, desmin residues were stained by immunofluorescence (green). (J) Transmission light microscopy and single immunofluorescence channels of (K) K18 (red) and (L) desmin (green) of the hepatocyte-like cell shown in I. Combined immunofluorescence analysis of (M) BSEP, (N) NTCP, and (0) K18 (red) with desmin (green) was also performed in HSCs cultured for 21 days without growth factor treatment (control). unexplained thus far. As shown in the present study, transplanted $\mathrm{eGFP}^{+}$HSCs can contribute to the formation of $\mathrm{K}^{+} 9^{+}$and $\alpha$-fetoprotein ${ }^{+}$cells during ductular reactions, indicating that MSCs can generate progenitor cells in vivo. Furthermore, isolated HSCs cultured on collagen can form tube-like structures, as reported earlier (16). We provided an explanation of the simultaneous expression of mesenchymal and epithelial proteins in progenitor cells by time-resolved hepatic differentiation experiments. During in vitro differentiation of HSCs, HSC clones, BM MSCs, and UCBSCs, all MSC populations shifted from a mesodermal to an endodermal expression pattern during differentiation into hepatocytes. In this intermediate stage, they transiently coexpressed mesenchymal and epithelial markers, as is known to occur with progenitor cells. In addition, we found the recently described hepatic stem cell marker LGR5 (37) to be transiently upregulated during hepatic differentiation of MSCs. These findings, on the one hand, are in line with the observed contribution of transplanted HSCs to ductular reactions as well as the occurrence of mesodermal protein residues (vimentin and desmin) in $\mathrm{K} 9^{+}$progenitor cells in vivo and, on the other hand, suggest a participation of MSC populations in progenitor cell formation and liver repair. Desmin ${ }^{+}$and nestin ${ }^{+}$cells that surround ductular structures with liver progenitor cells in the cen- ter (38) indicate the presence of MSCs. The present study shows that liver progenitor cells can originate from liver-resident stellate cells and other MSC populations that can potentially migrate from distant organs to the liver after severe injury in order to support regeneration. Thus, mesenchymal cells that surround ductular reactions may not only influence the differentiation of progenitor cells, as described recently (36), but may also contribute to their population. Evidence exists that hepatocyte and bile duct precursors originate from stem cells in the canals of Hering (39), and the ductular reaction emerges in the portal field. It remains an unresolved question whether MSC populations always contribute to progenitor cell formation or only under extreme conditions. Removal of HSCs from their niche in the space of Disse through enzymatic isolation and transplantation into the regenerating liver via the circulation creates an extreme situation that can promote their differentiation. Also, as shown in the present study, the injury model affects the outcome of the experiments. We found a higher engraftment of transplanted HSCs in the 2AAF/PHX model compared with that in the retrorsine/PHX model. The environment obviously controls the behavior of transplanted HSCs, which can respond in at least 3 ways: (a) HSCs remain activated and produce extracellular matrix and factors to influence neighboring cells; (b) 
activated HSCs can become quiescent again; and (c) HSCs differentiate into epithelial cells. The latter 2 possibilities may explain the absence of increased Col1a2, Col4a1, and Acta2 expression in the host liver despite the proliferation of transplanted HSCs. Regardless of the controversies emerging from recent lineage-tracing studies that argue for (17-19) or against $(20,21)$ a contribution of HSCs to liver regeneration, evidence for the characterization of HSCs as liver-resident stem cells and for their contribution to tissue repair was obtained in this study. These findings do not contradict those of previous studies demonstrating a contribution of HSCs to fibrogenesis, because chronic liver diseases certainly affect their behavior, as is also supposed for MSCs (40). With respect to mesenchymal and epithelial characteristics in progenitor cells, a recent study indicated a contribution of prominin $-1^{+}$/ $\mathrm{K}^{1} 9^{+}$liver progenitor cells with mesodermal markers to fibrosis in biliary atresia (41). Future research should address whether MSC populations are connected to stem cell niches within the canals of Hering and contribute to progenitor cell formation. Monitoring of hepatic differentiation of MSC populations and mesodermal proteins in epithelial cells in ductular reactions, as demonstrated in this study, as well as recent lineage-tracing experiments with the Acta2 promoter $(18,19)$, support this possibility.

\section{Methods}

Cell isolation and culture. A detailed description of cell isolation and culture as well as FACS protocols is provided in the Supplemental Methods.

Liver injury and HSC transplantation. Pellets that contained 2-acetylaminofluorene (70 mg, 2AAF 14-day release; Innovative Research of America) were implanted under the neck skin of WT Wistar rats (250 g) (Heinrich Heine University animal facility) 7 days before PHX was performed as previously described $(42,43)$. eGFP ${ }^{+}$HSCs from Wistar-TgN(CAG-GFP)184Ys rats (Rat Resource \& Research Center, University of Missouri, Columbia, Missouri, USA) were enriched by density gradient centrifugation (44), purified by retinoid-dependent FACS, and finally transplanted into WT female Wistar rats via tailvein injections immediately after PHX. About 700,000 $\pm 100,000$ HSCs or clonally expanded HSCs from male $\mathrm{eFFP}^{+}$rats were injected into each rat that underwent liver injury. HSCs were transplanted into a normal and a mutant Wistar rat strain (GUNN-UGT1A1//BluHsdRrrc; Rat Resource \& Research Center) (45). Homozygous mutants lack UGT1A1 enzyme activity and exhibit elevated bilirubin serum levels. Direct (conjugated) and indirect (unconjugated) bilirubin as well as albumin concentration in the serum of rats were determined by the Central Hospital Laboratory of Heinrich Heine University 4 weeks after HSC transplantation. For retransplantation experiments, FACS-sorted eGFP ${ }^{+}$HSCs were stained with $10 \mu \mathrm{M}$ CellTracker Red (C34552; Invitrogen) in suspension under serum-free conditions at $37^{\circ} \mathrm{C}$ for 45 minutes. After removal of excess dye by repeated centrifugation, about 100,000 double-labeled HSCs were transplanted into WT rats treated with 2AAF immediately after PHX. BM cells of the host animal were harvested 1 day after transplantation, cultured for 14 days, and then transplanted into other WT rats pretreated with $2 \mathrm{AAF}$ and PHX (about 10 million cells). In another injury model, female WT Wistar rats (250 g) received 2 i.p. retrorsine (R0382-100MG; SigmaAldrich) injections $(30 \mathrm{mg} / \mathrm{kg}) 14$ days apart. After an additional 4 weeks, PHX was performed (46), and FACS-sorted eGFP ${ }^{+}$HSCs from male Wistar rats were transplanted as described above.
Cell differentiation. The differentiation of isolated HSCs, HSC clones, BM MSCs, and UCBSCs was induced by $50 \mathrm{ng} / \mathrm{ml} \mathrm{FGF4} \mathrm{(100-}$ 31; PreproTech) and $40 \mathrm{ng} / \mathrm{ml}$ human HGF (100-39; PreproTech) dissolved in Iscove's modified Dulbecco's medium (12440-046; Invitrogen) supplemented with $1 \%$ insulin-transferrin-sodium selenite (ITS) (I1884; Sigma-Aldrich), 1\% linolic acid-albumin from bovine serum (L9530; Sigma-Aldrich), and 1\% antibiotic-antimycotic solution (Invitrogen). The differentiation medium was exchanged every second to third day. Cells in control experiments were cultured in the same medium, but without FGF4 and HGF. The progression of cell differentiation was monitored at weekly intervals.

Analysis of cells and tissue sections. Isolated cells and cryosections of the liver were fixed with either ice-cold methanol for 5 minutes or $4 \%$ formalin for 10 to 20 minutes and incubated with antibodies against GFAP (MAB3402; Millipore), K18 (BM2275P; Acris), K19 (NB100687; Novus Biologicals or 65129; ProGen Biotechnik), panK (CM162; Biocare Medical), desmin (ab8592; Abcam), vimentin (M0725; Dako), nestin (sc-33677; Santa Cruz Biotechnology Inc.), LGR5 (GTX62071; GeneTex), and HNF4 $\alpha$ (3113; Cell Signaling Technology) for immunofluorescence staining. The primary antibodies against NTCP and BSEP were provided by B. Stieger and P. Meier (University Hospital Zürich, Zürich, Switzerland). Appropriate secondary cyanine dye 3 (Су3) or FITC-labeled antibodies (AP182C, AP162C, AP192F, and AP182F; Millipore) and DAPI (P36935, ProLonged Gold; Invitrogen) were applied to mark primary antibodies and cell nuclei, respectively. Transplanted $\mathrm{eGFP}^{+}$HSCs were detected by immunohistochemistry on formalinfixed and Triton X-100-treated (0.1\% in PBS, 3051.3, Carl Roth GmbH) cryosections of the liver using monoclonal (2956, D5.1 XP; Cell Signaling Technology) and polyclonal antibodies (210-PS-1GFP; ImmunoKontact) against eGFP followed by labeling with secondary antibodies coupled to alkaline phosphatase or HRP (K5361, EnVision G|2 Double Stain System Rabbit/Mouse; Dako). Polyclonal eGFP antibodies and secondary antibodies used to detect eGFP were preincubated with liver homogenate from WT rats for at least 30 minutes to prevent unspecific binding. Fast red tablets containing levamisole for blocking endogenous alkaline phosphatase (11496549001; Roche) were used to prepare the substrate solution for alkaline phosphatase. Cell nuclei were counterstained by diluted hematoxylin solution (1:4 in distilled water) (1.05175; Merck) on fast red-stained liver sections. The hematoxylin solution was removed by washing with distilled and tap water. $\mathrm{eGFP}^{+}$cell types were identified by antibodies against HNF $4 \alpha, \mathrm{K} 19$, or desmin and subsequently labeled by appropriate secondary antibodies coupled to fluorescent dyes. For reverse transcriptase PCR (RT-PCR), tissue samples were taken from at least 4 different liver lobes, and the first-strand cDNA was made from $1 \mu \mathrm{g}$ total RNA per $20 \mu \mathrm{l}$ reaction volume using the RevertAid H Minus First Strand cDNA Synthesis Kit (K1632; Thermo Scientific). PCR was performed according to standard protocols using the 2xPCR Master Mix (K0171; Thermo Scientific), 0.8 $\mu \mathrm{mol} / \mathrm{l}$ primers (Supplemental Table 6), and 1 or $5 \mu$ template cDNA per $25 \mu \mathrm{l}$ reaction volume. A maximum of 20 or 35 cycles was used to amplify PCR products. The SensiMix SYBR No-ROX Kit (QT650-05; Bioline) was used for real-time qPCR according to the manufacturer's instructions. To assess the engraftment of male HSCs after transplantation, total DNA was purified with the DNeasy Blood \& Tissue Kit (69581; QIAGEN). For the amplification of PCR products, $12.5 \mathrm{ng}$ cDNA or 500 ng DNA and $0.6 \mu \mathrm{mol} / 1$ primers (Supplemental Table 7) were taken for each reaction. After an initial denaturation at $95^{\circ} \mathrm{C}$, 
the annealing was carried out at $58^{\circ} \mathrm{C}$, and elongation was performed at $72^{\circ} \mathrm{C}$ using 40 cycles and a TOptical Cycler (Analytik Jena AG). All samples were measured in triplicate, and hypoxanthine-guanine phosphoribosyltransferase 1 (Hprt1 mRNA of isolated cells) or $\beta$-actin (DNA of liver tissue) was used as a reference for the normalization of the results obtained by the $2^{-\Delta \Delta C t}$ method. Rat albumin was quantified in the culture supernatant by a rat-specific albumin ELISA (E110-125; Bethyl Laboratories) according to the manufacturer's recommendations. For the detection of UGT1A1 enzymes in the livers of normal and GUNN rats, the PCR product of Ugt1a1 mRNA was cleaved by the BstNI restriction enzyme (New England Biolabs) according to the manufacturer's instructions and separated by agarose gels (3\%). DNA probes for rat $\mathrm{Y}$ (IDRR1070; IDLabs) and X (IDRF1067; IDLabs) chromosomes were used for FISH analysis after fixation of liver sections with Carnoy's fixative for 15 minutes and tissue digestion by Pepsin (10108057001; Roche) for 5 minutes as previously described (24).

Analysis of bile acids and their conjugates. Bile acids were analyzed by UHPLC-MS/MS in culture supernatants of HGF- and FGF4-treated cells. The UHPLC-MS/MS system consisted of a UPLC-H class coupled to a Xevo TQ-S triple-quadruple mass spectrometer (Waters). Electrospray ionization was performed in the negative ionization mode. Chromatographic separation was performed on a UPLC HSS C18 column (Waters) $(2.1 \times 100 \mathrm{~mm}, 1.8 \mu \mathrm{m})$. The mobile phase consisted of water containing $0.1 \%$ formic acid and $5 \mathrm{mM}$ ammonium acetate (eluent A) and acetonitrile (eluent B). Analytes were separated by a gradient elution. The injection volume was $5 \mu \mathrm{l}$, and the column was maintained at $40^{\circ} \mathrm{C}$. Detection of the bile acids and their glycine and taurine conjugates was performed in selected reaction monitoring (SRM) mode. All standards as well as the deuterated internal standards (IS) substance (d4-CA, d4-GCA, and d4-GCDCA) were purchased from Sigma-Aldrich and Steraloids.
Statistics. The significance of the data obtained by rat albumin ELISA and qPCR was determined by ANOVA or Mann-Whitney $U$ test, and data were considered significant at a $P$ value of less than 0.05 . The results of at least 3 independent experiments were expressed as mean values in percentages. The variance was specified as the mean \pm SEM.

Study approval. All animal procedures were approved by the relevant federal authority for animal protection (Landesamt für Natur, Umwelt und Verbraucherschutz Nordrhein-Westfalen, Recklinghausen, Germany; reference numbers 9.93.2.10.34.07.163 and 84-02.04.2012.A344), and the animals received care according to the German animal welfare act.

\section{Acknowledgments}

The authors are grateful to Claudia Rupprecht (Clinic of Gastroenterology, Hepatology and Infectious Diseases) and Katharina Raba (Institute of Transplantation Diagnostics and Cell Therapeutics) of the Heinrich Heine University for expert technical assistance. We thank the Rat Resource \& Research Center (P40OD011062) for providing GUNN rats and eGFP-expressing rats. Transgenic eGFP ${ }^{+}$ rats were generated by Eiji Kobayashi (Center for Development of Advanced Medical Technology, Jichi Medical University, Tochigi, Japan). This work was supported by the German Research Foundation (DFG) through the Collaborative Research Center SFB 974 ("Communication and Systems Relevance during Liver Injury and Regeneration,” Düsseldorf, Germany).

Address correspondence to: Dieter Häussinger, Clinic of Gastroenterology, Hepatology and Infectious Diseases, Heinrich Heine University Düsseldorf, Moorenstraße 5, 40225 Düsseldorf, Germany. Phone: 004.211.81.17569; E-mail: haeussin@ uni-duesseldorf.de.
1. Friedman SL, Roll FJ, Boyles J, Bissell DM. Hepatic lipocytes: the principal collagen-producing cells of normal rat liver. Proc Natl Acad Sci US A. 1985;82(24):8681-8685.

2. Friedman SL. Hepatic stellate cells: protean, multifunctional, and enigmatic cells of the liver. Physiol Rev. 2008;88(1):125-172.

3. Geerts A. On the origin of stellate cells: mesodermal, endodermal or neuro-ectodermal? J Hepatol. 2004;40(2):331-334.

4. Gard A, White F, Dutton G. Extra-neural glial fibrillary acidic protein (GFAP) immunoreactivity in perisinusoidal stellate cells of rat liver. J Neuroimmunol. 1985;8(4-6):359-375.

5. Enzan H, Himeno H, Hiroi M, Kiyoku H, Saibara T, Onishi S. Development of hepatic sinusoidal structure with special reference to the Ito cells. Microsc Res Tech. 1997;39(4):336-349.

6. Asahina K, et al. Mesenchymal origin of hepatic stellate cells, submesothelial cells, and perivascular mesenchymal cells during mouse liver development. Hepatology. 2009;49(3):998-1011.

7. Yokoi Y, et al. Immunocytochemical detection of desmin in fat-storing cells (Ito cells). Hepatology. 1984;4(4):709-714.

8. Kordes C, Sawitza I, Götze S, Häussinger D. Hepatic stellate cells support hematopoiesis and are liver-resident mesenchymal stem cells. Cell Physiol Biochem. 2013;31(2-3):290-304.
9. Castilho-Fernandes A, et al. Human hepatic stellate cell line (LX-2) exhibits characteristics of bone marrow-derived mesenchymal stem cells. Exp Mol Pathol. 2011;91(3):664-672.

10. Baba S, et al. Commitment of bone morrow cells to hepatic stellate cells in mouse. J Hepatol. 2004;40(2):255-260.

11. Russo FP, et al. The bone marrow functionally contributes to liver fibrosis. Gastroenterology. 2006;130(6):1807-1821.

12. Friedenstein AJ, Chailakhjan RK, Lalykina KS. The development of fibroblast colonies in monolayer cultures of guinea-pig bone marrow and spleen cells. Cell Tissue Kinet. 1970;3(4):393-403.

13. Crisan M, et al. A perivascular origin for mesenchymal stem cells in multiple human organs. Cell Stem Cell. 2008;3(3):301-313.

14. Sawitza I, Kordes C, Reister S, Häussinger D. The niche of stellate cells within rat liver. Hepatology. 2009;50(5):1617-1624.

15. Kordes C, Häussinger D. Hepatic stem cell niches. J Clin Invest. 2013;123(5):1874-1880.

16. Kordes C, et al. CD133+ hepatic stellate cells are progenitor cells. Biochem Biophys Res Commun. 2007;352(2):410-417.

17. Yang L, et al. Fate-Mapping evidence that hepatic stellate cells are epithelial progenitors in adult mouse livers. Stem Cells. 2008;26(8):2104-2113.

18. Michelotti GA, et al. Smoothened is a master regulator of adult liver repair. JClin Invest. 2013;123(6):2380-2394.

19. Swiderska-Syn M, et al. Myofibroblastic cells function as progenitors to regenerate murine livers after partial hepatectomy. Gut. 2014;63(8):1333-1344.

20. Mederacke I, et al. Fate tracing reveals hepatic stellate cells as dominant contributors to liver fibrosis independent of its aetiology. Nat Commun. 2013;4:2823.

21. Lua I, et al. Mesodermal mesenchymal cells give rise to myofibroblasts, but not epithelial cells, in mouse liver injury. Hepatology. 2014;60(1):311-322.

22. Orsolits B, et al. Retinoid machinery in distinct neural stem cell populations with different retinoid responsiveness. Stem Cells Dev. 2013;22(20):2777-9273.

23. David R, et al. Selection of a common multipotent cardiovascular stem cell using the $3.4-\mathrm{kb}$ MesP1 promoter fragment. Basic Res Cardiol. 2013;108(1):312.

24. Kordes C, Sawitza I, Götze S, Häussinger D. Stellate cells from rat pancreas are stem cells and can contribute to liver regeneration. PLoS One. 2012;7(12):e51878.

25. Sato Y, et al. Human mesenchymal stem cells xenografted directly to rat liver are differentiated into human hepatocytes without fusion. Blood. 2005;106(2):756-763. 
26. Chamberlain J, et al. Efficient generation of human hepatocytes by the intrahepatic delivery of clonal human mesenchymal stem cells in fetal sheep. Hepatology. 2007;46(6):1935-1945.

27. Aurich I, et al. Functional integration of hepatocytes derived from human mesenchymal stem cells into mouse livers. Gut. 2007;56(3):405-415.

28. Smith A. Cell therapy: In search of pluripotency. Curr Biol. 1998;8(22):R802-R804.

29. Shafritz DA. Stem cells and liver repopulation: Current reality and prospects for the future. In: Okita K, ed. Stem Cells And Liver Regeneration. Tokyo, Japan: Springer Japan; 2004:41-50.

30. Camberlain G, Fox J, Ashton B, Middleton J. Mesenchymal stem cells: their phenotype, differentiation capacity, immunological features, and potential for homing. Stem Cells. 2007;25(11):2739-2749.

31. Fong LSE, Chan CK, Goodman SB. Stem cell homing in musculoskeletal injury. Biomaterials. 2011;32(2):395-409.

32. Michalopoulos GK. Principles of liver regeneration and growth homeostasis. Compr Physiol. 2013;3(1):485-513.
33. Riehle KJ, Dan YY, Campbell JS, Fausto N. New concepts in liver regeneration. J Gastroenterol Hepatol. 2011;26(suppl 1):203-212.

34. Schmelzer E, et al. Human hepatic stem cells from fetal and postnatal donors. J Exp Med. 2007;204(8):1973-1987.

35. Haque S, et al. Identification of bipotential progenitor cells in human liver regeneration. Lab Invest. 1996;75(5):699-705.

36. Boulter L, et al. Macrophage-derived Wnt opposes Notch signaling to specify hepatic progenitor cell fate in chronic liver disease. Nat Med. 2012;18(4):572-579.

37. Huch M, et al. In vitro expansion of single Lgr5 $5^{+}$ liver stem cells induced by Wnt-driven regeneration. Nature. 2013;494(7436):247-250.

38. Reister S, Kordes C, Sawitza I, Häussinger D. The epigenetic regulation of stem cell factors in hepatic stellate cells. Stem Cells Dev. 2011;20(10):1687-1699.

39. Kuwahara R, et al. The hepatic stem cell niche: identification by label-retaining cell assay. Hepatology. 2008;47(6):1994-2002.

40. Lepperdinger G. Inflammation and mes- enchymal stem cell aging. Curr Opin Immunol. 2011;23(4):518-524.

41. Mavila N, et al. Expansion of PROMININ-1expressing cells in association with fibrosis of biliary atresia. Hepatology. 2014;60(3):941-953.

42. Higgins GM, Anderson RM. Experimental pathology of the liver. I. Restoration of the liver of the white rat following partial surgical removal. Arch Pathol Lab Med.1931;12:186-202.

43. Tatematsu M, et al. Studies on the proliferation and fate of oval cells in the liver of rats treated with 2-acetylaminofluorene and partial hepatectomy. Am J Pathol. 1984;114(3):418-430.

44. Hendriks HFJ, Verhoofstad WA, Brouwer A, de Leeuw AM, Knook DL. Perisinusoidal fatstoring cells are the main vitamin A storage sites in rat liver. Exp Cell Res. 1985;160(1):138-149.

45. Gunn $\mathrm{CH}$. Hereditary acholuric jaundice in a new mutant strain of rats. J Hered. 1938;29(4):137-139.

46. Gordon GJ, Coleman WB, Hixson DC, Grisham JW. Liver regeneration in rats with retrorsineinduced hepatocellular injury proceeds through a novel cellular response. Am J Pathol. 2000;156(2):607-619. 